Supporting Information for

\title{
Unsymmetrical PNP-Pincer Type Phosphaalkene Ligands Protected by a Fused-Ring Bulky Eind Group: Synthesis and Applications to $\operatorname{Rh}(I)$ and $\operatorname{Ir}(I)$ Complexes
}

\author{
Hiro-omi Taguchi, ${ }^{\dagger}$ Daichi Sasaki, ${ }^{\dagger}$ Katsuhiko Takeuchi, ${ }^{\dagger}$ Shota Tsujimoto, ${ }^{\ddagger}$ Tsukasa Matsuo,${ }^{\ddagger}$ \\ Hiromasa Tanaka, ${ }^{\S}$ Kazunari Yoshizawa ${ }^{\S}$ and Fumiyuki Ozawa*,† \\ ${ }^{\dagger}$ International Research Center for Elements Science (IRCELS), Institute for Chemical Research, \\ Kyoto University, Uji, Kyoto 611-0011, Japan \\ *Department of Applied Chemistry, Faculty of Science and Engineering, Kinki University, \\ Higashi-Osaka, Osaka 577-8502, Japan \\ ${ }^{\S}$ Institute for Materials Chemistry and Engineering, International Research Center for Molecular \\ Systems, Kyushu University, Nishi-ku, Fukuoka 819-0395, Japan \\ e-mail: ozawa@scl.kyoto-ua.c.jp
}

\section{Contents}

Table S1. Crystal Data and Details of Structure Determination for 8 and 9

Table S2. Crystal Data and Details of Structure Determination

for $[\mathrm{K}(18 \mathrm{C} 6)][\mathbf{1 2}]$ and $[\mathrm{K}(18 \mathrm{C} 6)][\mathbf{1 3}]$

Table S3. Crystal Data and Details of Structure Determination for $\mathbf{1 4}$

Figure S1. X-Ray structure of $\left[\mathrm{Rh}\left(\mathrm{NH}_{3}\right)(\right.$ Eind-PPEP*) $)$ (14).

Figure S2. Optimized structure of $\left[\mathrm{Rh}\left(\mathrm{NH}_{3}\right)(\right.$ Eind-PPEP*)].

Figure S3. Optimized structure of $\left[\mathrm{Rh}\left(\mathrm{NH}_{2}\right)\right.$ (Eind-PPEP)].

Figure S4. Optimized structure of $\left[\operatorname{Ir}\left(\mathrm{NH}_{3}\right)(\right.$ Eind-PPEP*)].

Figure S5. Optimized structure of $\left[\operatorname{Ir}\left(\mathrm{NH}_{2}\right)(\right.$ Eind-PPEP)].

Figures S6-S35. NMR spectra of 6-15. 
Table S1. Crystal Data and Details of Structure Determination for $\mathbf{8}$ and $\mathbf{9}$

\begin{tabular}{|c|c|c|}
\hline Complex & 8 & 9 \\
\hline Empirical formula & $\mathrm{C}_{53} \mathrm{H}_{79} \mathrm{ClNP}_{2} \mathrm{Rh}$ & $\mathrm{C}_{53} \mathrm{H}_{79} \mathrm{ClIrNP}_{2}$ \\
\hline Formula weight & 930.47 & 1019.76 \\
\hline$T(\mathrm{~K})$ & $103(2)$ & $103(2)$ \\
\hline Crystal system & Orthorhombic & Orthorhombic \\
\hline Space group & $P 2{ }_{1} 2_{1}$ & $P 2_{1} 2_{1} 2_{1}$ \\
\hline$a(\AA)$ & $14.904(4)$ & $14.9689(18)$ \\
\hline$b(\AA)$ & $17.034(5)$ & $16.995(2)$ \\
\hline$c(\AA)$ & $19.351(6)$ & $19.372(2)$ \\
\hline$\alpha\left(^{\circ}\right)$ & 90 & 90 \\
\hline$\beta\left(^{\circ}\right)$ & 90 & 90 \\
\hline$\gamma\left({ }^{\circ}\right)$ & 90 & 90 \\
\hline$V\left(\AA^{3}\right)$ & $4913(3)$ & $4928.4(10)$ \\
\hline$Z$ & 4 & 4 \\
\hline$d_{\text {calc }}\left(\mathrm{g} / \mathrm{cm}^{3}\right)$ & 1.258 & 1.374 \\
\hline$\mu(\operatorname{Mo~K} \alpha)\left(\mathrm{mm}^{-1}\right)$ & 0.502 & 2.863 \\
\hline$F(000)$ & 1984 & 2112 \\
\hline Crystal size & $0.10 \times 0.01 \times 0.01$ & $0.14 \times 0.10 \times 0.06$ \\
\hline$\theta$ range $\left(^{\circ}\right)$ & 1.59 to 24.50 & 2.10 to 27.50 \\
\hline Reflections collected & 39728 & 50475 \\
\hline Independent reflections $\left(R_{\text {int }}\right)$ & $8182(0.1482)$ & $11301(0.0183)$ \\
\hline Absorption correction & Multi-scan & Multi-scan \\
\hline Max and min transmission & 0.9950 and 0.9515 & 1.0000 and 0.9386 \\
\hline Data / restraints / parameters & 8182 / 0 / 540 & $11301 / 0 / 540$ \\
\hline GOF on $F^{2}$ & 1.072 & 1.074 \\
\hline$R 1, w R 2[I>2 \sigma(I)]$ & $0.0796,0.1918$ & $0.0198,0.0440$ \\
\hline$R 1, w R 2$ (all data) & $0.1108,0.2333$ & $0.0211,0.0591$ \\
\hline Absolute structure parameter & $0.04(6)$ & $-0.017(4)$ \\
\hline Largest peak and hole (e $\AA^{3}$ ) & 2.043 and -0.984 & 1.506 and -0.437 \\
\hline
\end{tabular}


Table S2. Crystal Data and Details of Structure Determination for $[\mathrm{K}(18 \mathrm{C} 6)][\mathbf{1 2}]$ and $[\mathrm{K}(18 \mathrm{C} 6)][\mathbf{1 3}]$

\begin{tabular}{|c|c|c|}
\hline Complex & {$[\mathrm{K}(18 \mathrm{C} 6)][\mathbf{1 2}]$} & {$[\mathrm{K}(18 \mathrm{C} 6)][\mathbf{1 3}]$} \\
\hline Empirical formula & $\mathrm{C}_{65} \mathrm{H}_{102} \mathrm{ClKNO}_{6} \mathrm{P}_{2} \mathrm{Rh}$ & $\mathrm{C}_{65} \mathrm{H}_{102} \mathrm{ClIrKNO}_{6} \mathrm{P}_{2}$ \\
\hline Formula weight & 1232.88 & 1322.17 \\
\hline$T(\mathrm{~K})$ & $103(2)$ & $103(2)$ \\
\hline Crystal system & Monoclinic & Monoclinic \\
\hline Space group & $P 2_{1} / c$ & $P 2_{1} / c$ \\
\hline$a(\AA)$ & $12.1391(13)$ & $12.222(3)$ \\
\hline$b(\AA)$ & $29.372(3)$ & $29.346(8)$ \\
\hline$c(\AA)$ & $18.861(2)$ & $18.875(5)$ \\
\hline$\alpha\left(^{\circ}\right)$ & 90 & 90 \\
\hline$\beta\left({ }^{\circ}\right)$ & $104.2756(14)$ & 104.227(3) \\
\hline$\gamma\left({ }^{\circ}\right)$ & 90 & 90 \\
\hline$V\left(\AA^{3}\right)$ & $6517.2(12)$ & $6562(3)$ \\
\hline$Z$ & 4 & 4 \\
\hline$d_{\text {calc }}\left(\mathrm{g} / \mathrm{cm}^{3}\right)$ & 1.257 & 1.338 \\
\hline$\mu(\operatorname{Mo~K} \alpha)\left(\mathrm{mm}^{-1}\right)$ & 0.464 & 2.235 \\
\hline$F(000)$ & 2632 & 2760 \\
\hline Crystal size & $0.19 \times 0.07 \times 0.05$ & $0.11 \times 0.05 \times 0.01$ \\
\hline$\theta$ range $\left(^{\circ}\right)$ & 1.73 to 27.50 & 1.72 to 25.00 \\
\hline Reflections collected & 78774 & 54836 \\
\hline Independent reflections $\left(R_{\text {int }}\right)$ & $14941(0.0477)$ & $11540(0.0929)$ \\
\hline Absorption correction & Multi-scan & Multi-scan \\
\hline Max and min transmission & 0.9772 and 0.9170 & 1.0000 and 0.7957 \\
\hline Data / restraints / parameters & $14941 / 0 / 710$ & $11540 / 0 / 710$ \\
\hline GOF on $F^{2}$ & 1.160 & 1.159 \\
\hline$R 1, w R 2[I>2 \sigma(I)]$ & $0.0474,0.1190$ & $0.0511,0.1183$ \\
\hline$R 1, w R 2$ (all data) & $0.0570,0.1321$ & $0.0692,0.1404$ \\
\hline Largest peak and hole (e $\left.\AA^{3}\right)$ & 1.387 and -1.117 & 1.927 and -1.179 \\
\hline
\end{tabular}


Table S3. Crystal Data and Details of Structure Determination for $\mathbf{1 4}$

\begin{tabular}{ll|ll}
\hline Empirical formula & $\mathrm{C}_{53} \mathrm{H}_{81} \mathrm{~N}_{2} \mathrm{P}_{2} \mathrm{Rh}$ & Crystal size & $0.09 \times 0.04 \times 0.03$ \\
Formula weight & 911.05 & $\theta$ range $\left(^{\circ}\right)$ & 2.40 to 25.50 \\
$T(\mathrm{~K})$ & $103(2)$ & Reflections collected & 49193 \\
Crystal system & Monoclinic & Independent reflections $\left(R_{\text {int }}\right)$ & $9102(0.1155)$ \\
Space group & $P 2_{1} / a$ & Absorption correction & Multi-scan \\
$a(\AA)$ & $15.5387(11)$ & Max and min transmission & 0.9866 and 0.9606 \\
$b(\AA)$ & $10.1599(7)$ & Data / restraints / parameters & $9102 / 0 / 515$ \\
$c(\AA)$ & $31.327(2)$ & GOF on $F^{2}$ & 1.384 \\
$\alpha\left(^{\circ}\right)$ & 90 & $R 1, w R 2[I>2 \sigma(I)]$ & $0.1458,0.3088$ \\
$\beta\left({ }^{\circ}\right)$ & $98.745(4)$ & $R 1, w R 2($ all data $)$ & $0.1601,0.3172$ \\
$\gamma\left({ }^{\circ}\right)$ & 90 & Largest peak and hole $\left(\mathrm{e} \AA^{3}\right)$ & 1.289 and -3.357 \\
$V\left(\AA^{3}\right)$ & $4888.2(6)$ & \\
$Z$ & 4 & & \\
$d_{\text {calc }}\left(\mathrm{g} / \mathrm{cm}^{3}\right)$ & 1.238 & & \\
$\mu(\mathrm{Mo} \mathrm{K} \alpha)\left(\mathrm{mm}^{-1}\right)$ & 0.451 & 1952 & \\
$F(000)$ & & \\
\hline
\end{tabular}

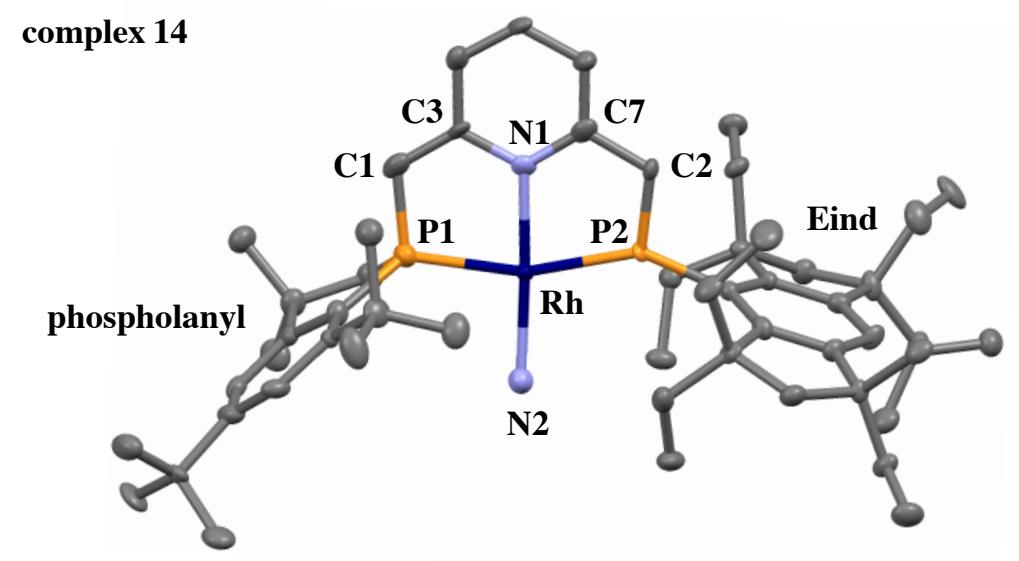

Figure S1. X-Ray structure of $\left[\mathrm{Rh}_{\left(\mathrm{NH}_{3}\right)(\text { Eind-PPEP*) }}\right.$ (14) with 50\% probability ellipsoids. Hydrogen atoms are omitted for clarity. Selected bond distances $(\AA)$ and angles $(\mathrm{deg}): \mathrm{Rh}-\mathrm{P} 1$ 2.274(3), Rh-P2 2.206(3), Rh-N1 2.058(10), Rh-N2 2.101(11), P1-C1 1.739(14), P2-C2 1.648(12), C1-C3 1.385(19), C2-C7 1.461(16), P1-Rh-P2 162.16(12), N1-Rh-N2 177.2(4). 


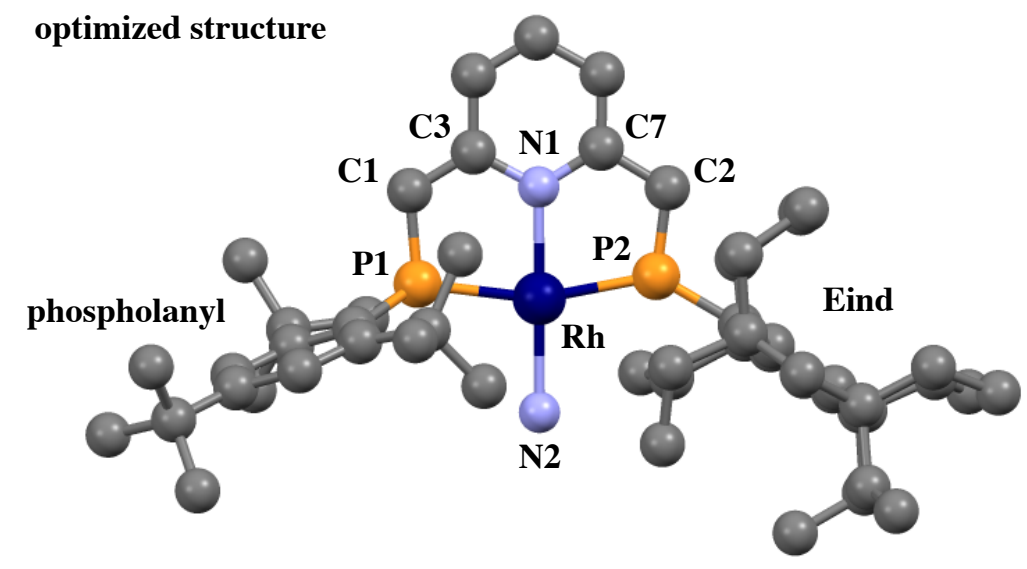

Figure S2. Optimized structure of $\left[\mathrm{Rh}\left(\mathrm{NH}_{3}\right)(\right.$ Eind-PPEP*)]. Hydrogen atoms are omitted for clarity. Selected bond distances $(\AA)$ and angles (deg): Rh-P1 2.302, Rh-P2 2.231, Rh-N1 2.101, Rh-N2 2.149, P1-C1 1.758, P2-C2 1.681, C1-C3 1.394, C2-C7 1.451, P1-Rh-P2 162.28, $\mathrm{N} 1-\mathrm{Rh}-\mathrm{N} 2178.81$.

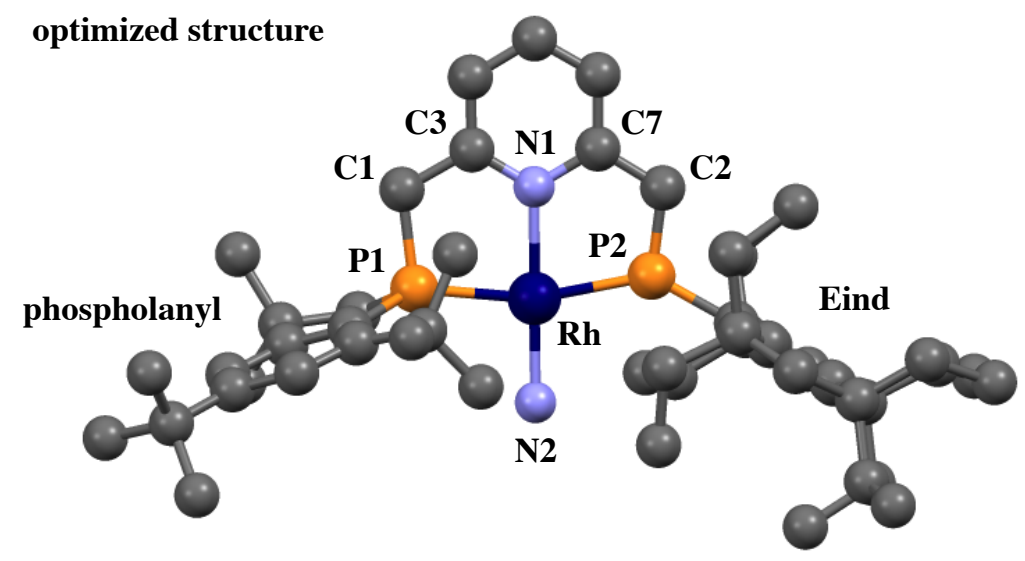

Figure S3. Optimized structure of $\left[\mathrm{Rh}\left(\mathrm{NH}_{2}\right)\right.$ (Eind-PPEP)]. Hydrogen atoms are omitted for clarity. Selected bond distances $(\AA)$ and angles (deg): Rh-P1 2.265, Rh-P2 2.236, Rh-N1 2.119, Rh-N2 1.970, P1-C1 1.876, P2-C2 1.695, C1-C3 1.513, C2-C7 1.428, P1-Rh-P2 164.21, $\mathrm{N} 1-\mathrm{Rh}-\mathrm{N} 2178.86$. 


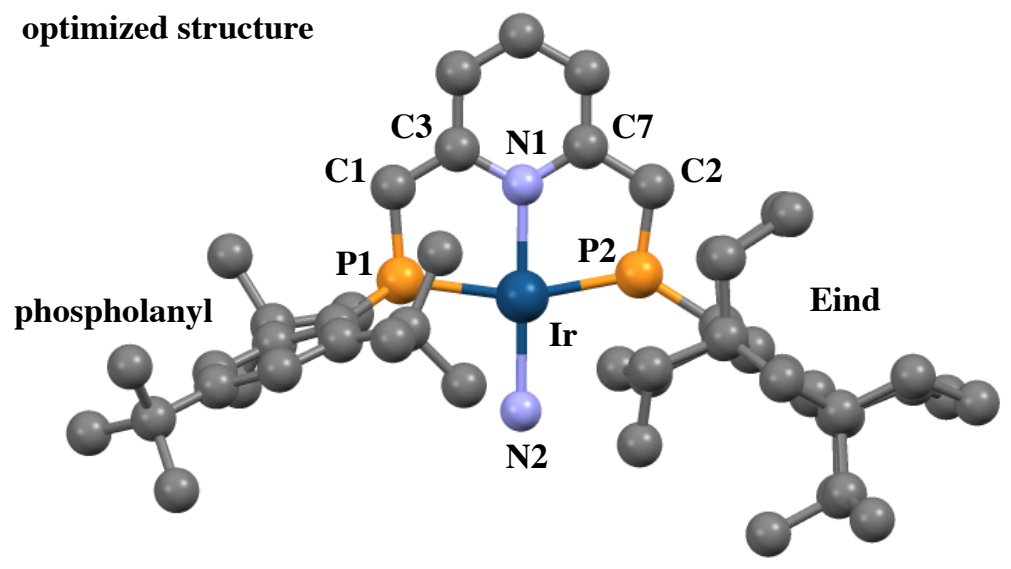

Figure S4. Optimized structure of $\left[\operatorname{Ir}\left(\mathrm{NH}_{3}\right)\right.$ (Eind-PPEP*)]. Hydrogen atoms are omitted for clarity. Selected bond distances $(\AA)$ and angles (deg): Ir-P1 2.305, Ir-P2 2.227, Ir-N1 2.103, IrN2 2.165, P1-C1 1.755, P2-C2 1.690, C1-C3 1.395, C2-C7 1.450, P1-Ir-P2 161.64, N1-Ir-N2 178.62.

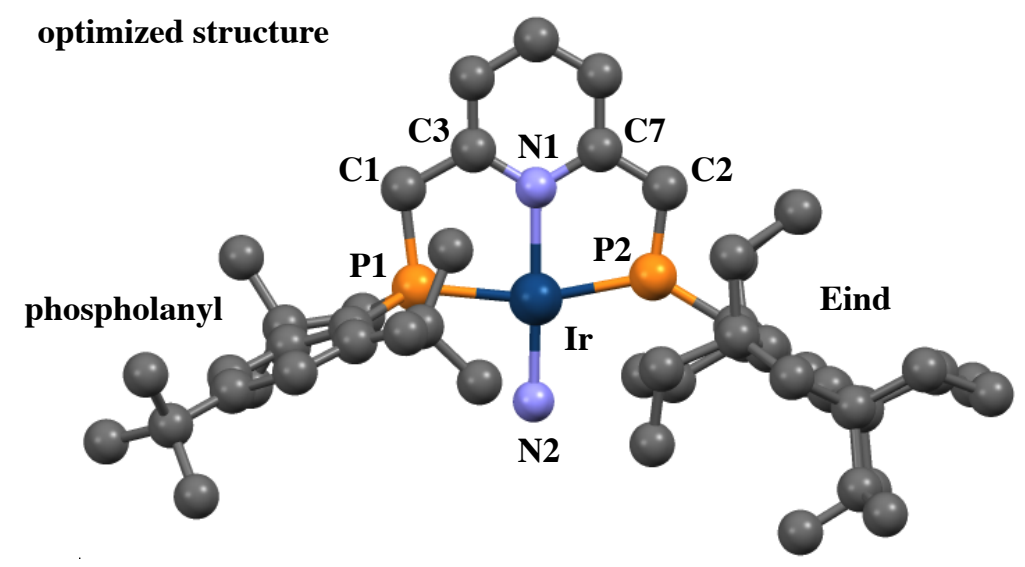

Figure S5. Optimized structure of $\left[\operatorname{Ir}\left(\mathrm{NH}_{2}\right)(\right.$ Eind-PPEP) $]$. Hydrogen atoms are omitted for clarity. Selected bond distances $(\AA)$ and angles (deg): Ir-P1 2.265, Ir-P2 2.237, Ir-N1 2.116, Ir$\mathrm{N} 2$ 1.970, P1-C1 1.870, P2-C2 1.697, C1-C3 1.512, C2-C7 1.422, P1-Ir-P2 162.75, $\mathrm{N} 1-\mathrm{Ir}-\mathrm{N} 2$ 177.15. 



Figure S6. ${ }^{1} \mathrm{H}$ NMR spectrum of Eind-PNO $(6)\left(\mathrm{C}_{6} \mathrm{D}_{6}, 25^{\circ} \mathrm{C}, 400 \mathrm{MHz}\right)$. 


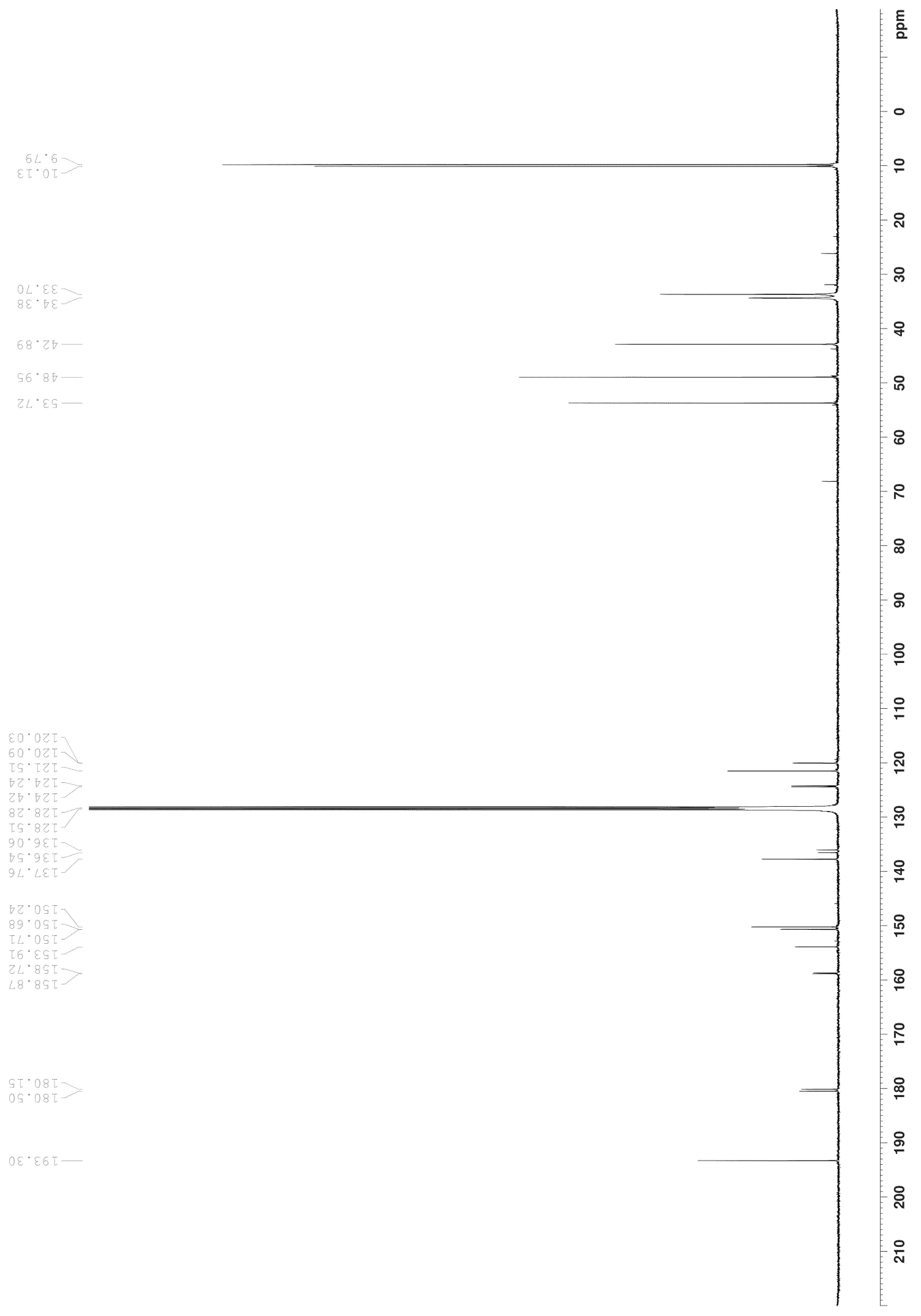

Figure S7. ${ }^{13} \mathrm{C}\left\{{ }^{1} \mathrm{H}\right\}$ NMR spectrum of Eind-PNO $(6)\left(\mathrm{C}_{6} \mathrm{D}_{6}, 25{ }^{\circ} \mathrm{C}, 101 \mathrm{MHz}\right)$. 


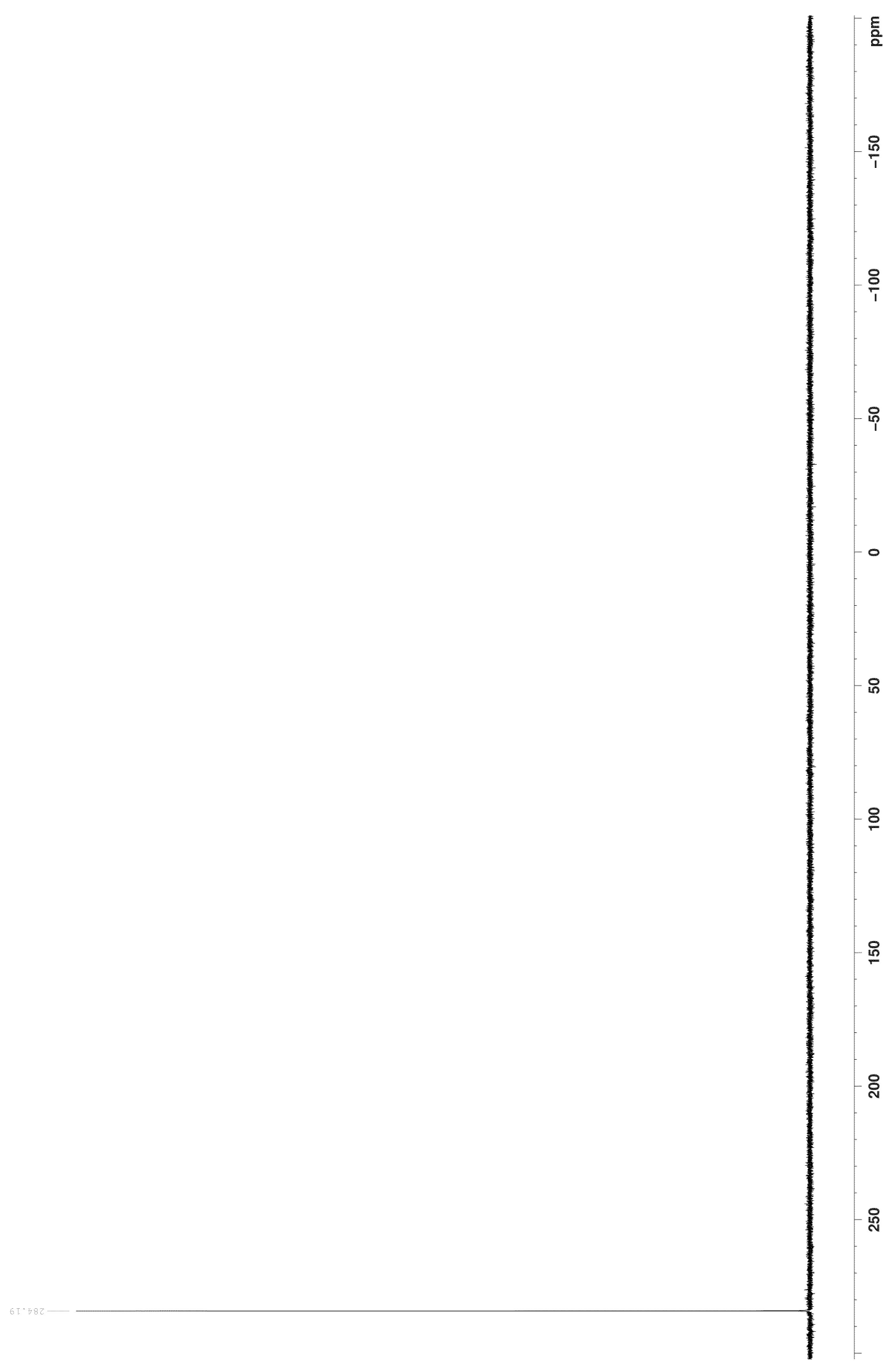

Figure S8. ${ }^{31} \mathrm{P}\left\{{ }^{1} \mathrm{H}\right\}$ NMR spectrum of Eind-PNO $(6)\left(\mathrm{C}_{6} \mathrm{D}_{6}, 25{ }^{\circ} \mathrm{C}, 162 \mathrm{MHz}\right)$. 

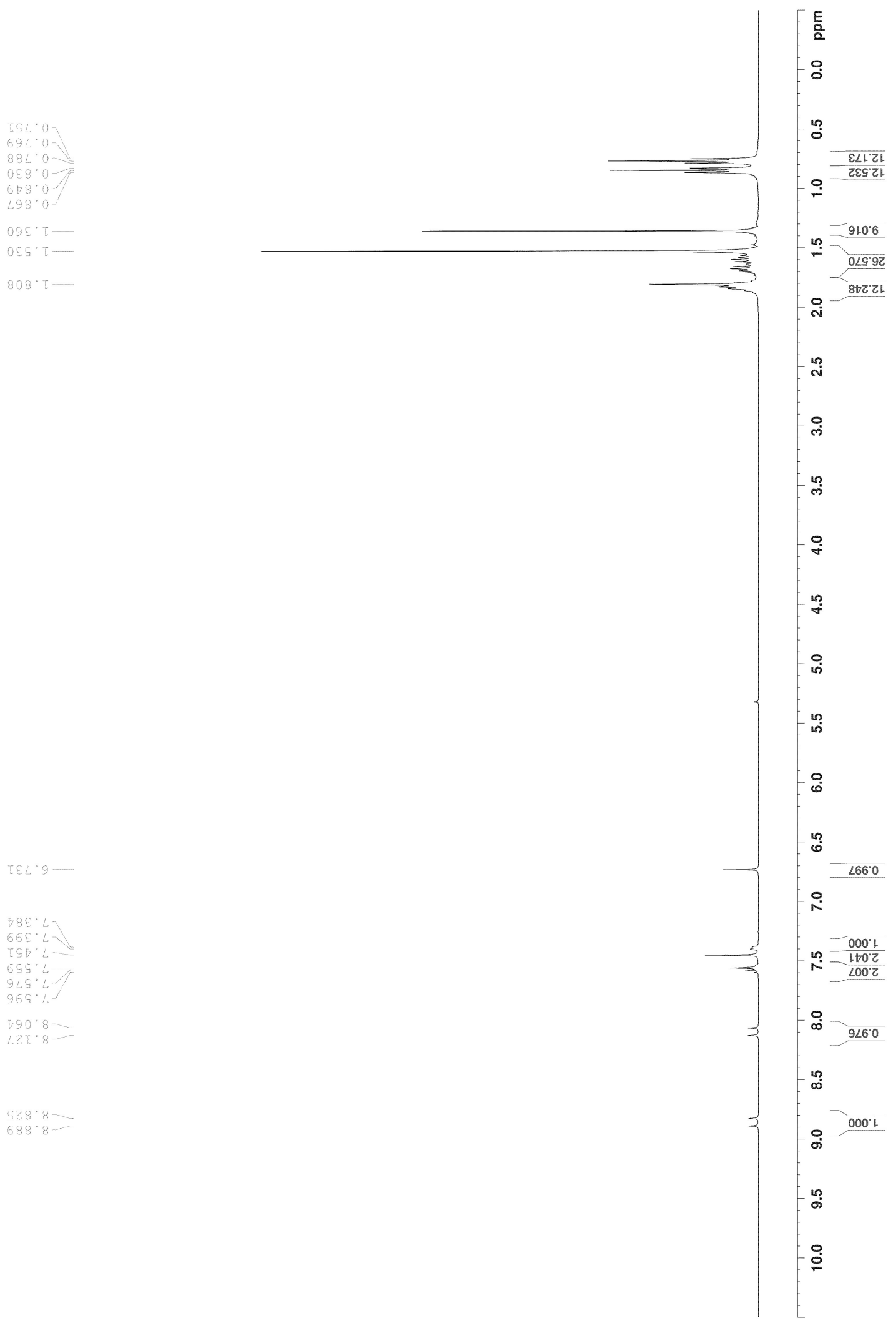

Figure S9. ${ }^{1} \mathrm{H}$ NMR spectrum of Eind-BPEP (7) $\left(\mathrm{CD}_{2} \mathrm{Cl}_{2}, 25{ }^{\circ} \mathrm{C}, 400 \mathrm{MHz}\right)$. 


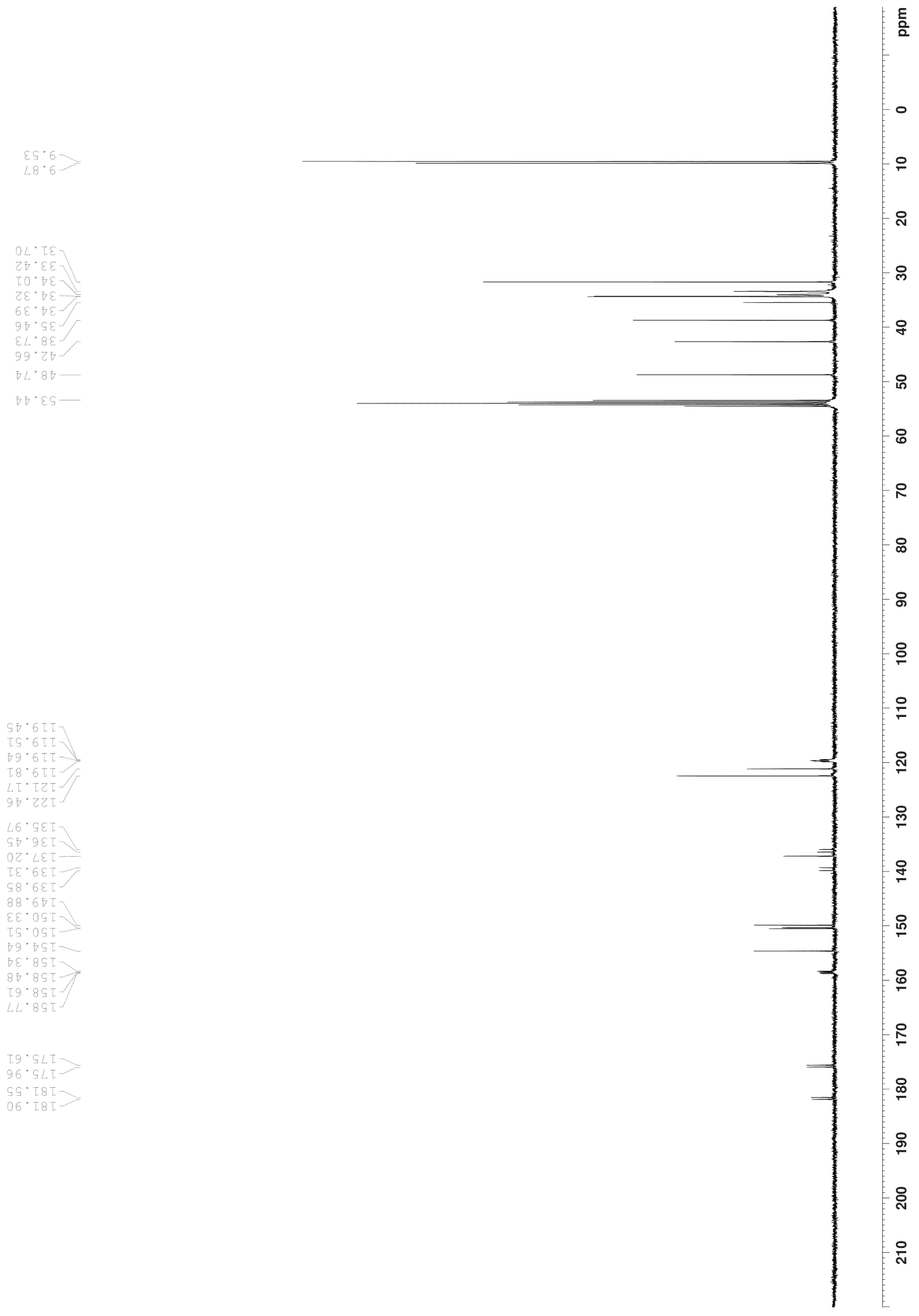

Figure S10. ${ }^{13} \mathrm{C}\left\{{ }^{1} \mathrm{H}\right\}$ NMR spectrum of Eind-BPEP (7) $\left(\mathrm{CD}_{2} \mathrm{Cl}_{2}, 25{ }^{\circ} \mathrm{C}, 101 \mathrm{MHz}\right)$. 


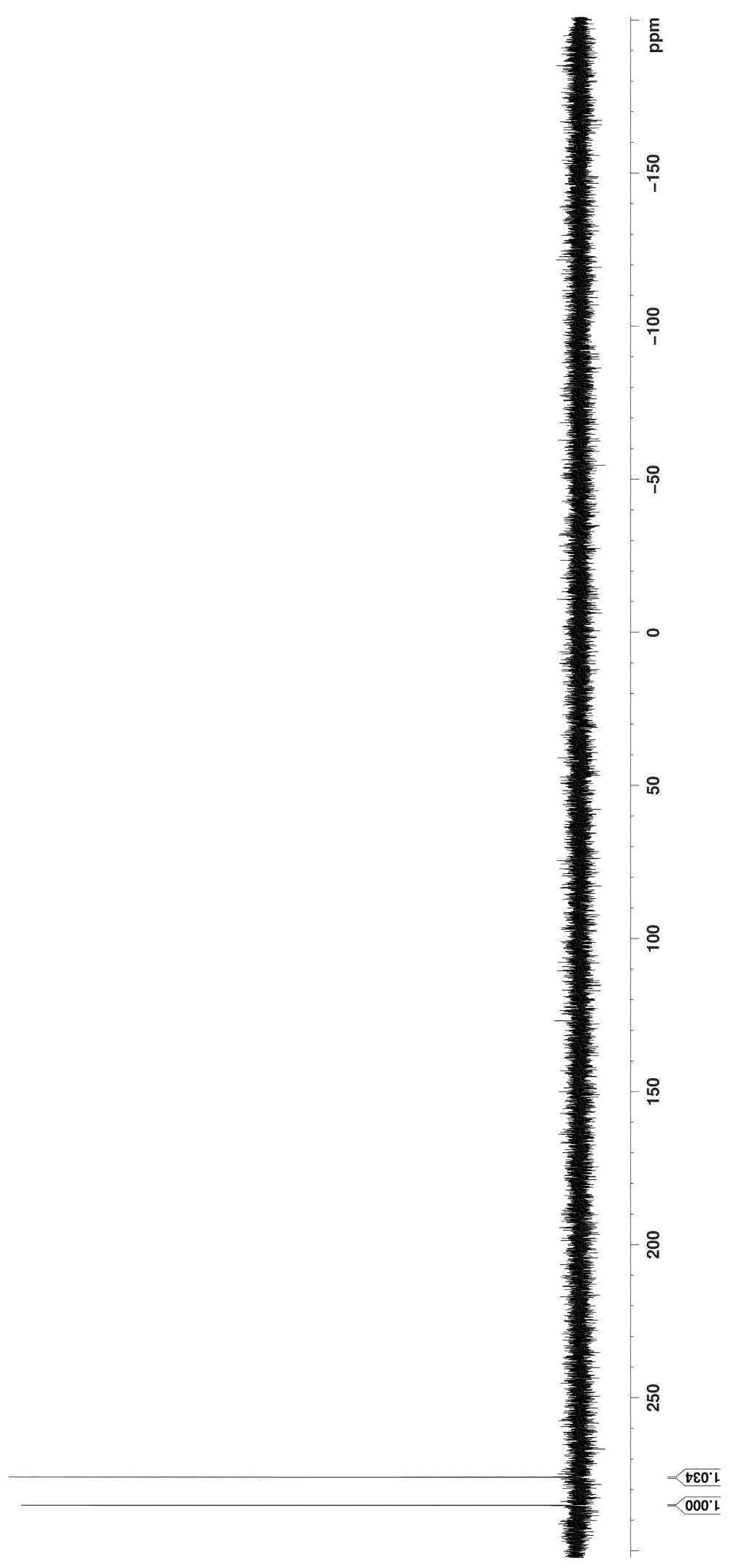

Figure S11. ${ }^{31} \mathrm{P}\left\{{ }^{1} \mathrm{H}\right\}$ NMR spectrum of Eind-BPEP $(7)\left(\mathrm{CD}_{2} \mathrm{Cl}_{2}, 25{ }^{\circ} \mathrm{C}, 162 \mathrm{MHz}\right)$. 

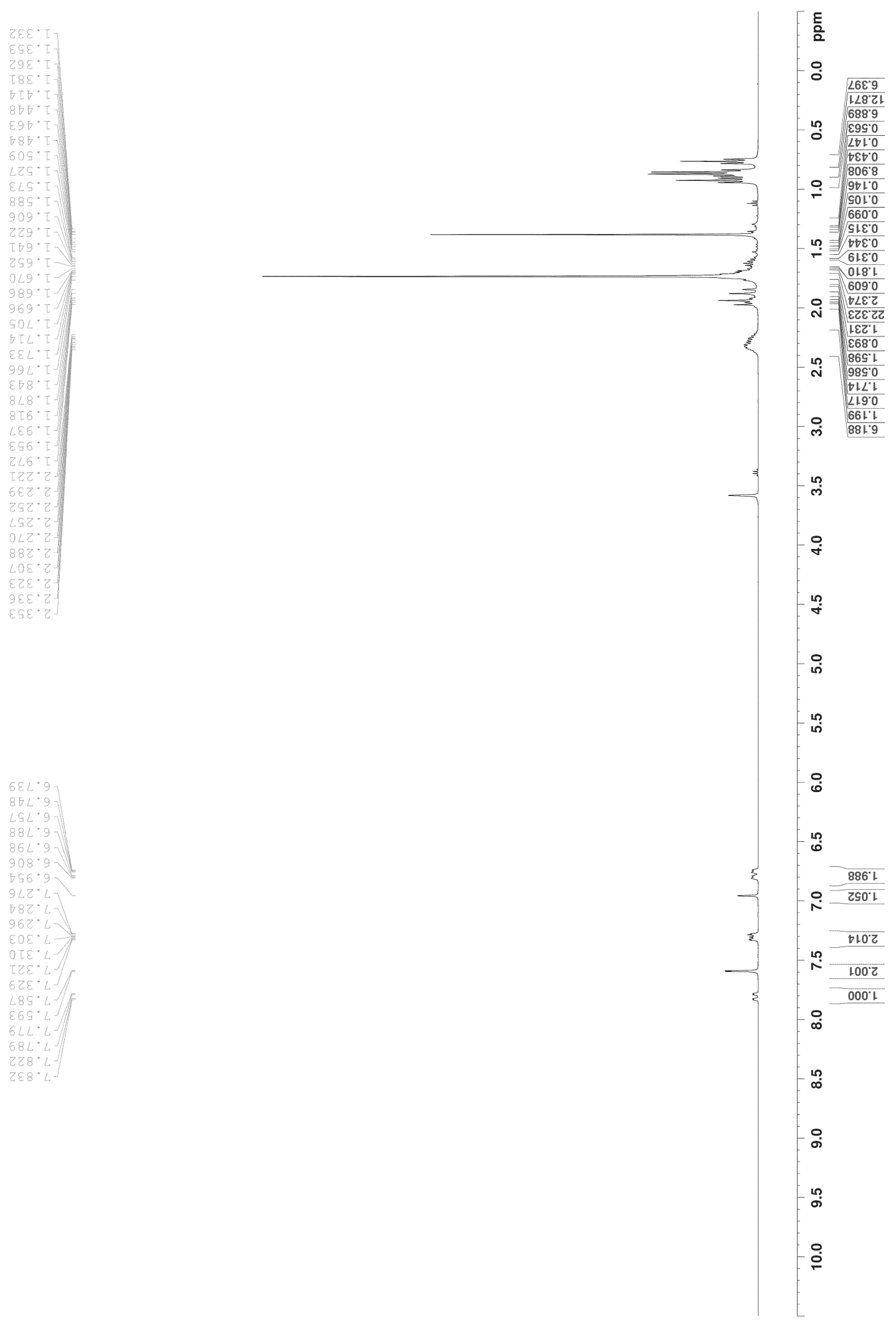

Figure S12. ${ }^{1} \mathrm{H} \mathrm{NMR}$ spectrum of $\left[\mathrm{RhCl}(\right.$ Eind-BPEP) $](8)\left(\mathrm{CD}_{2} \mathrm{Cl}_{2}, 25{ }^{\circ} \mathrm{C}, 400 \mathrm{MHz}\right)$. 


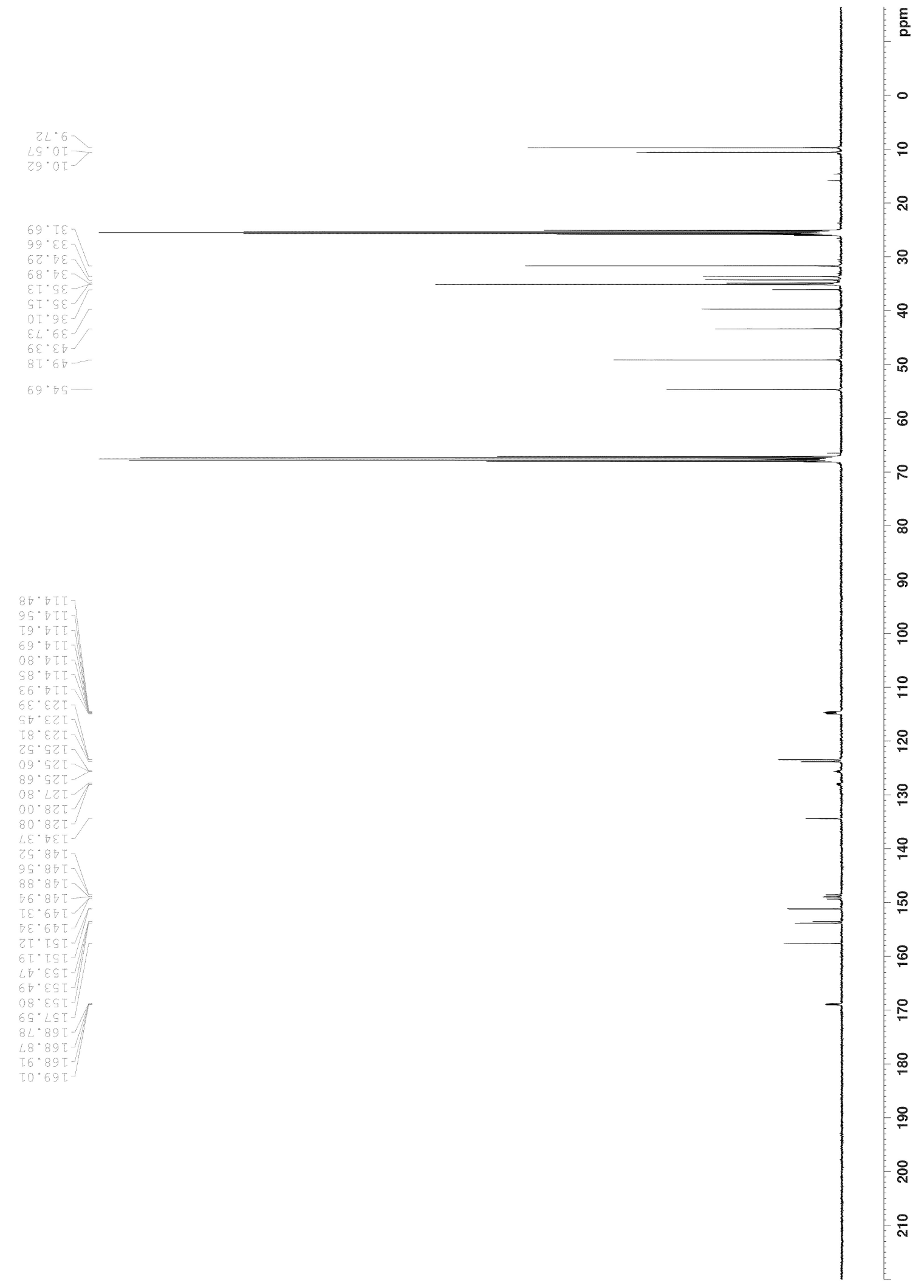

Figure S13. ${ }^{13} \mathrm{C}\left\{{ }^{1} \mathrm{H}\right\}$ NMR spectrum of $\left[\mathrm{RhCl}(\right.$ Eind-BPEP) $](8)\left(\mathrm{CD}_{2} \mathrm{Cl}_{2}, 25{ }^{\circ} \mathrm{C}, 101 \mathrm{MHz}\right)$. 


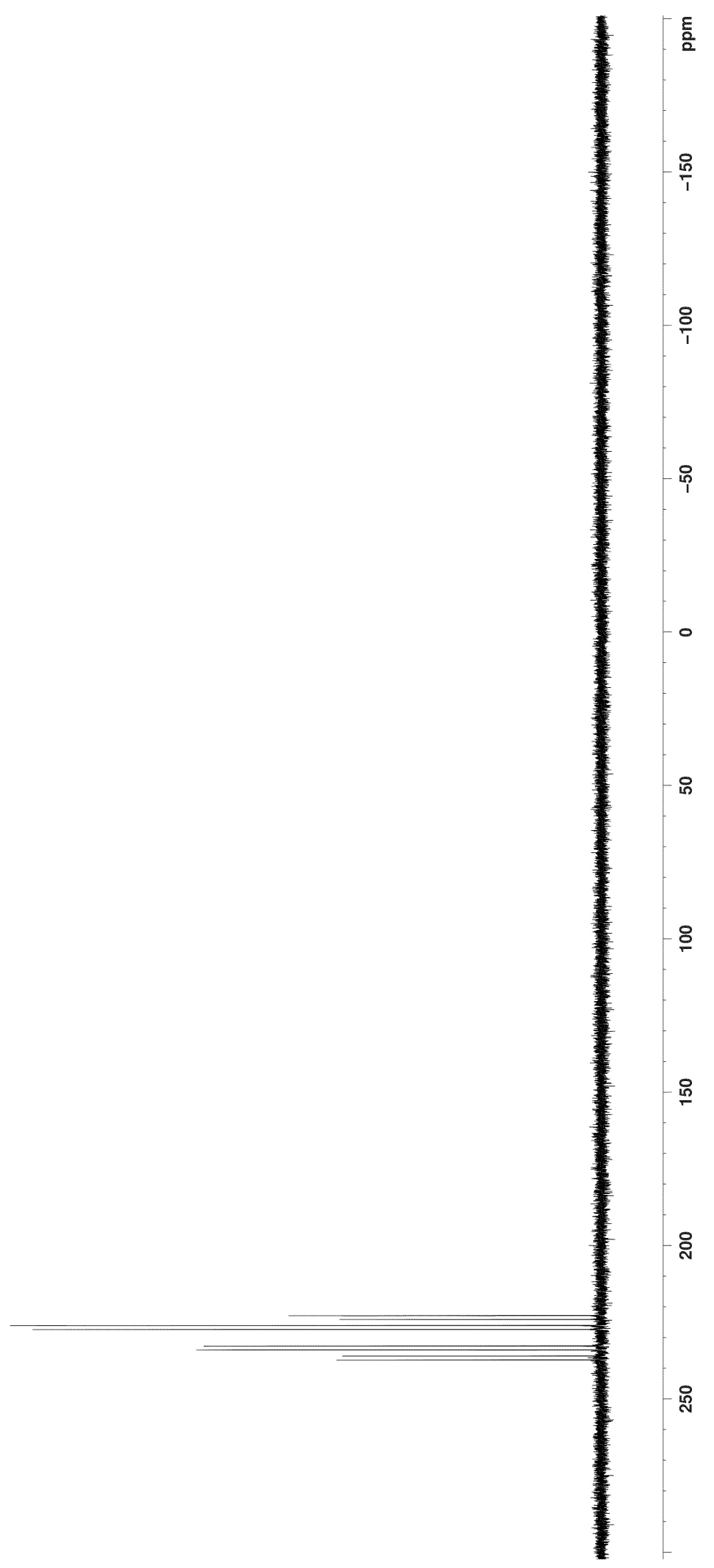

Figure S14. ${ }^{31} \mathrm{P}\left\{{ }^{1} \mathrm{H}\right\}$ NMR spectrum of $[\mathrm{RhCl}($ Eind-BPEP $)](8)\left(\mathrm{CD}_{2} \mathrm{Cl}_{2}, 25{ }^{\circ} \mathrm{C}, 162 \mathrm{MHz}\right)$. 

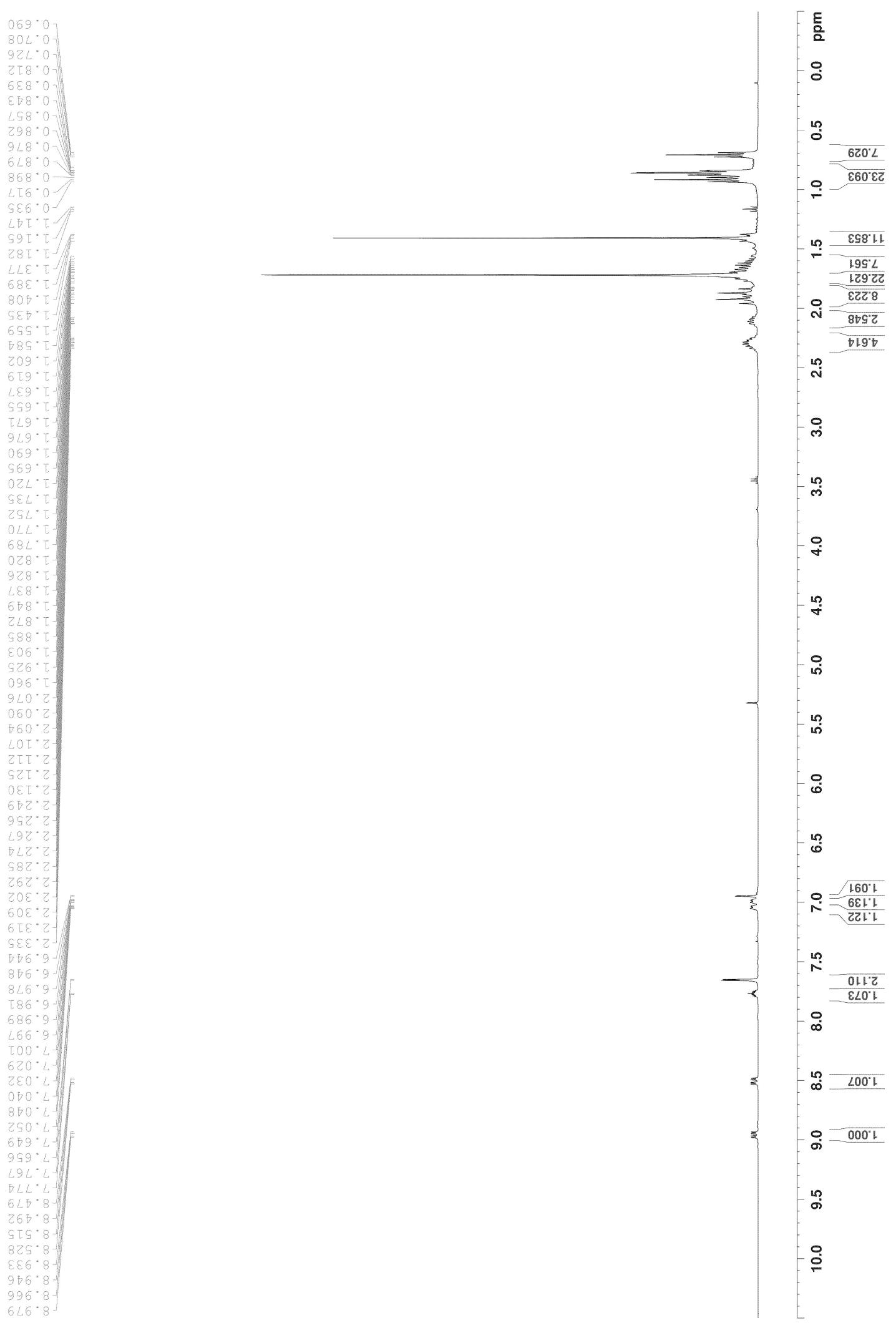

Figure S15. ${ }^{1} \mathrm{H} \mathrm{NMR}$ spectrum of $\left[\mathrm{IrCl}(\right.$ Eind-BPEP) $](9)\left(\mathrm{CD}_{2} \mathrm{Cl}_{2}, 25{ }^{\circ} \mathrm{C}, 400 \mathrm{MHz}\right)$. 


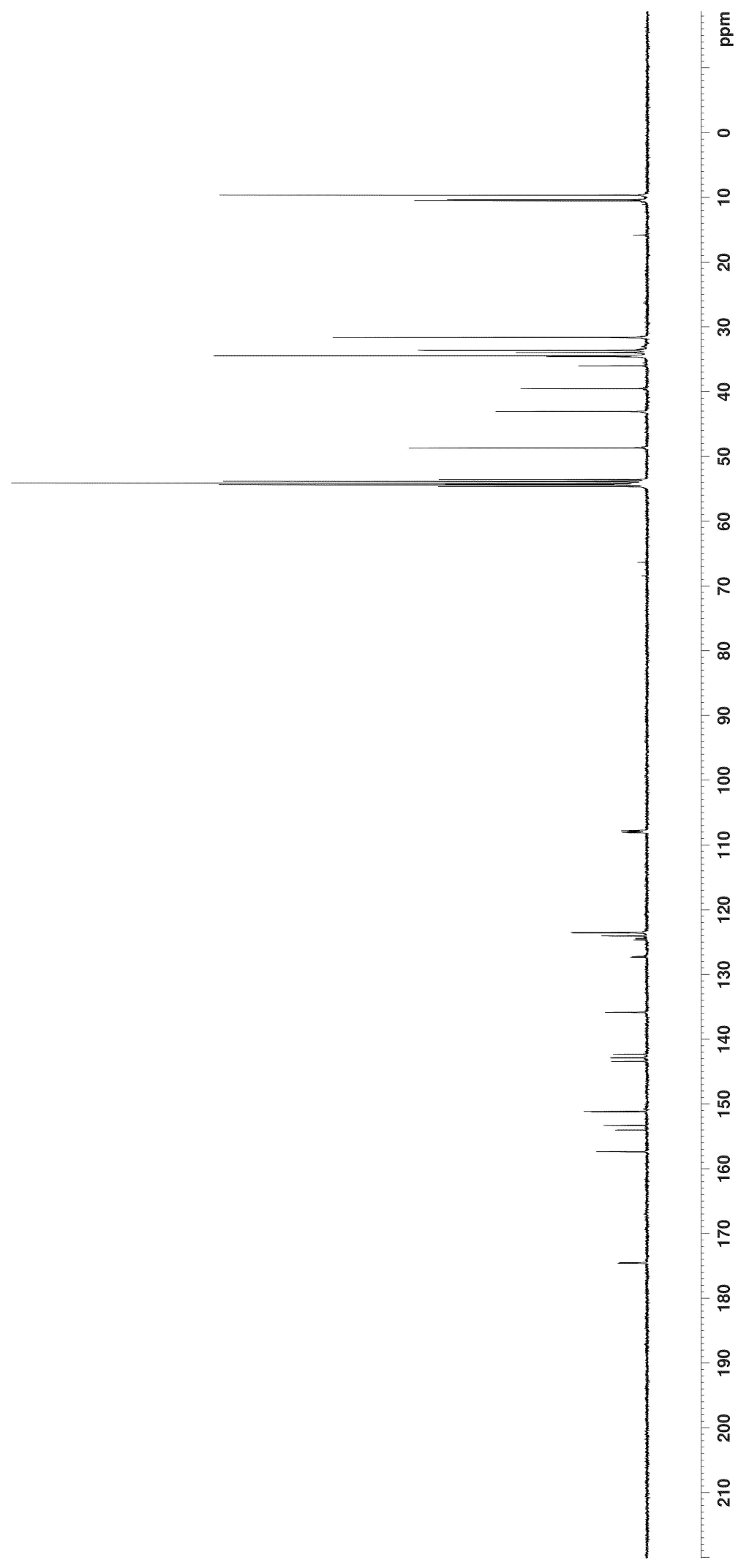

Figure S16. ${ }^{13} \mathrm{C}\left\{{ }^{1} \mathrm{H}\right\}$ NMR spectrum of $[\operatorname{IrCl}($ Eind-BPEP $)](9)\left(\mathrm{CD}_{2} \mathrm{Cl}_{2}, 25{ }^{\circ} \mathrm{C}, 101 \mathrm{MHz}\right)$. 


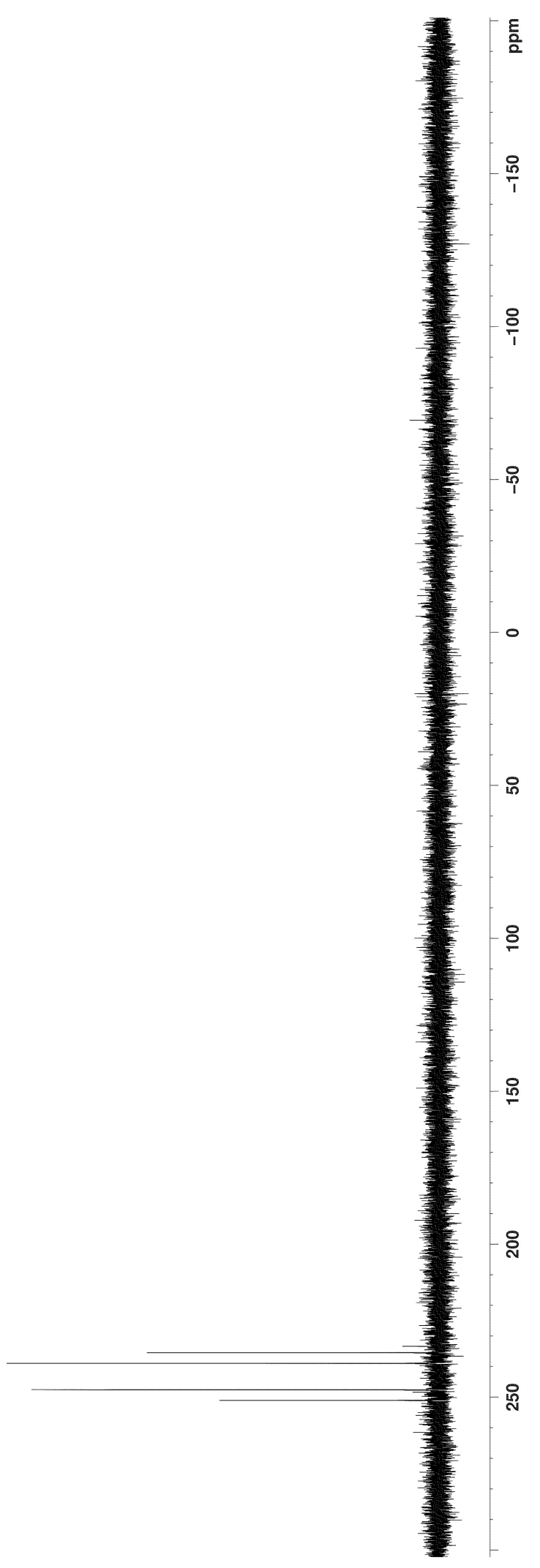

Figure S17. ${ }^{31} \mathrm{P}\left\{{ }^{1} \mathrm{H}\right\}$ NMR spectrum of $\left[\mathrm{IrCl}(\right.$ Eind-BPEP) $](9)\left(\mathrm{CD}_{2} \mathrm{Cl}_{2}, 25^{\circ} \mathrm{C}, 162 \mathrm{MHz}\right)$. 

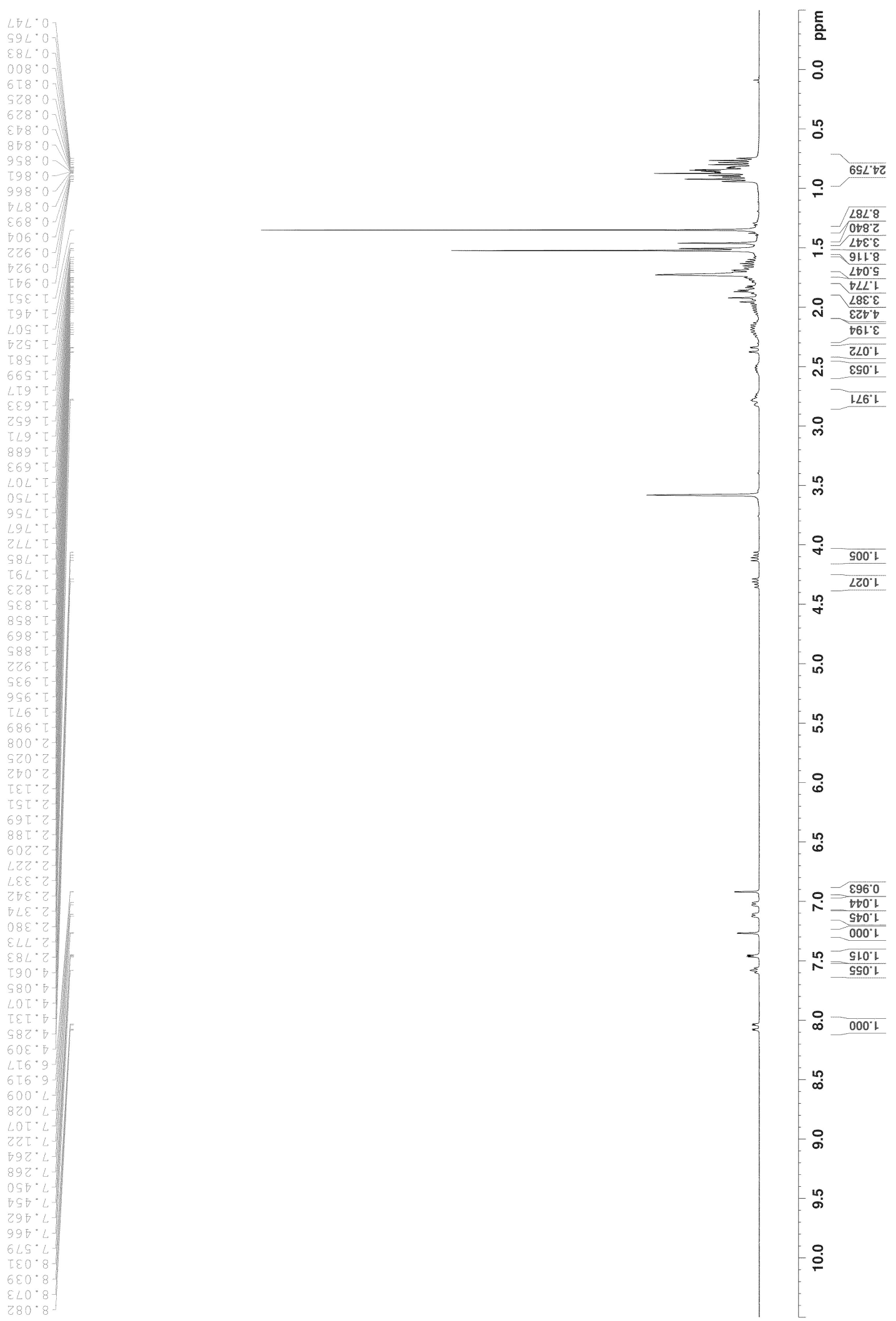

Figure S18. ${ }^{1} \mathrm{H}$ NMR spectrum of $\left[\mathrm{RhCl}(\right.$ Eind-PPEP) $](\mathbf{1 0})\left(\mathrm{THF}-d_{8}, 25^{\circ} \mathrm{C}, 400 \mathrm{MHz}\right)$. 


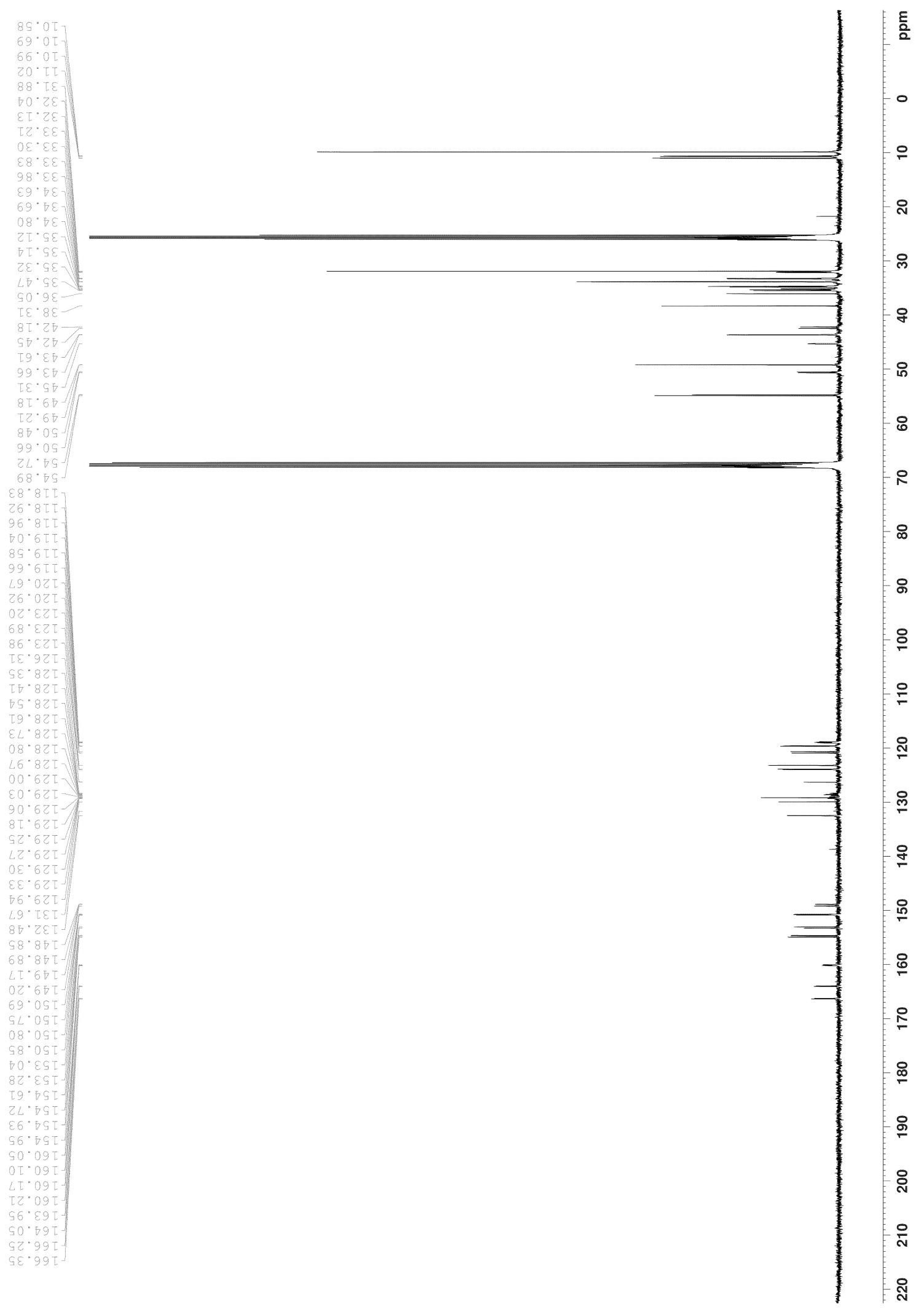

Figure S19. ${ }^{13} \mathrm{C}\left\{{ }^{1} \mathrm{H}\right\}$ NMR spectrum of $\left[\mathrm{RhCl}(\right.$ Eind-PPEP) $](\mathbf{1 0})\left(\mathrm{THF}-d_{8}, 25{ }^{\circ} \mathrm{C}, 101 \mathrm{MHz}\right)$. 


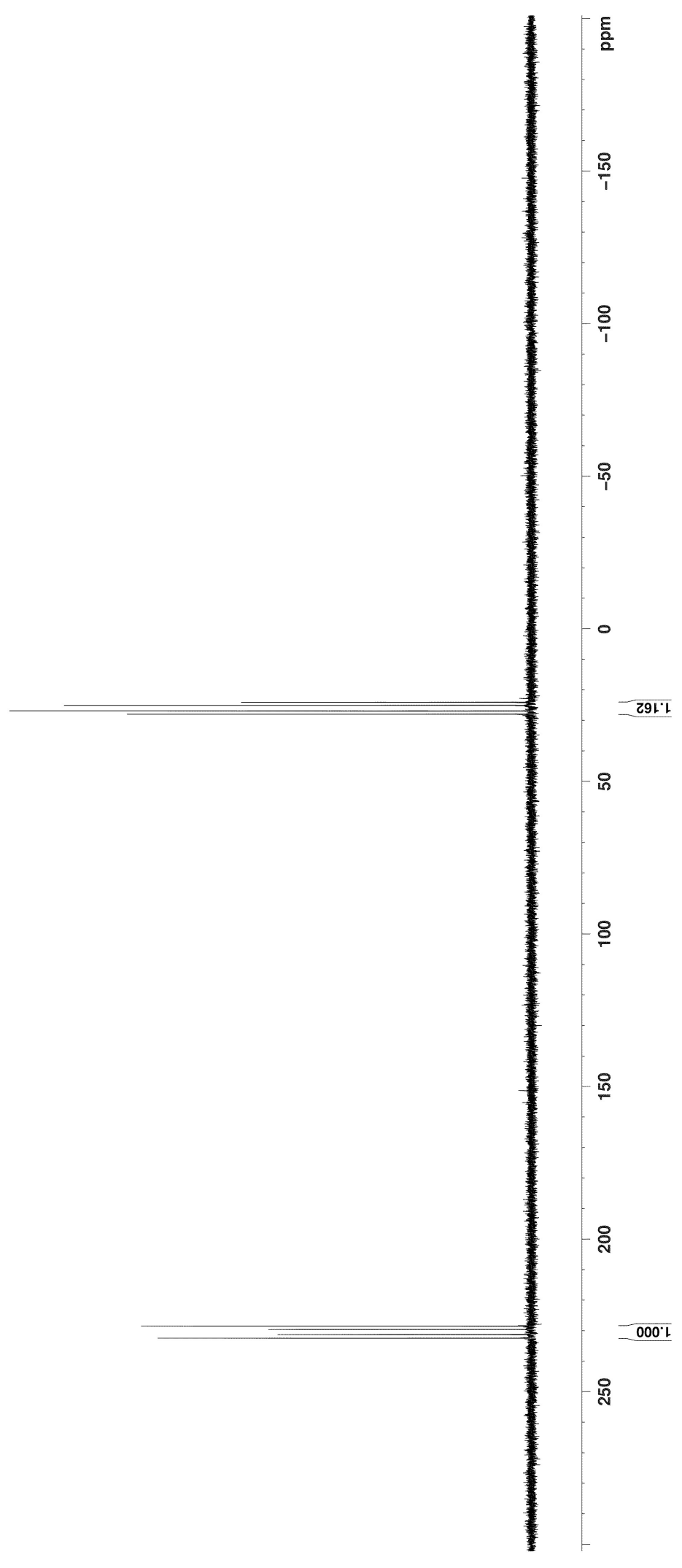

Figure S20. ${ }^{31} \mathrm{P}\left\{{ }^{1} \mathrm{H}\right\}$ NMR spectrum of $\left[\mathrm{RhCl}(\right.$ Eind-PPEP) $](\mathbf{1 0})\left(\mathrm{THF}-d_{8}, 25{ }^{\circ} \mathrm{C}, 162 \mathrm{MHz}\right)$. 


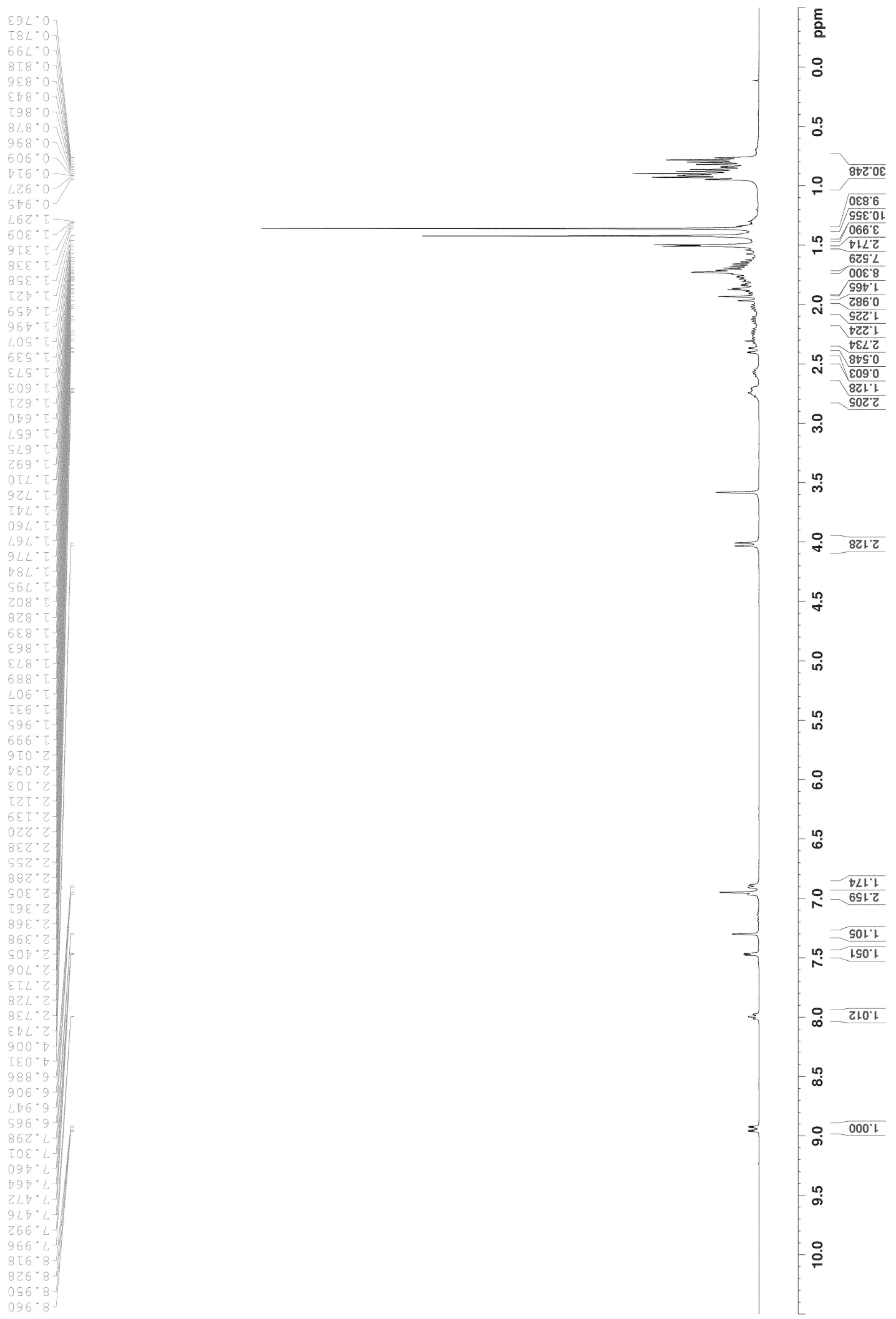

Figure S21. ${ }^{1} \mathrm{H}$ NMR spectrum of $\left[\operatorname{IrCl}(\right.$ Eind-PPEP) $](11)\left(\mathrm{THF}-d_{8}, 25{ }^{\circ} \mathrm{C}, 400 \mathrm{MHz}\right)$. 


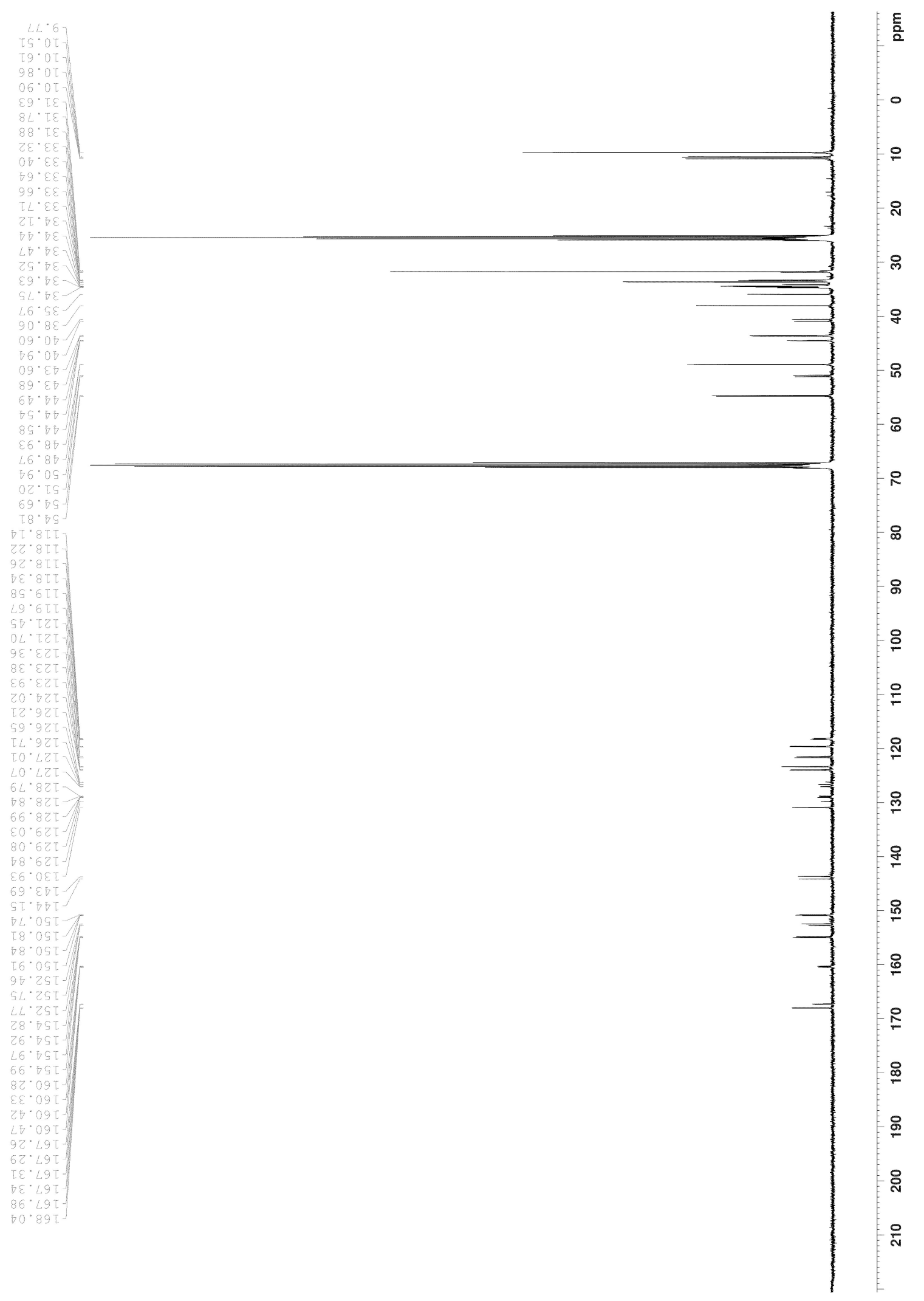

Figure S22. ${ }^{13} \mathrm{C}\left\{{ }^{1} \mathrm{H}\right\}$ NMR spectrum of [ $\operatorname{IrCl}($ Eind-PPEP) $](11)\left(\mathrm{THF}-d_{8}, 25{ }^{\circ} \mathrm{C}, 101 \mathrm{MHz}\right)$. 


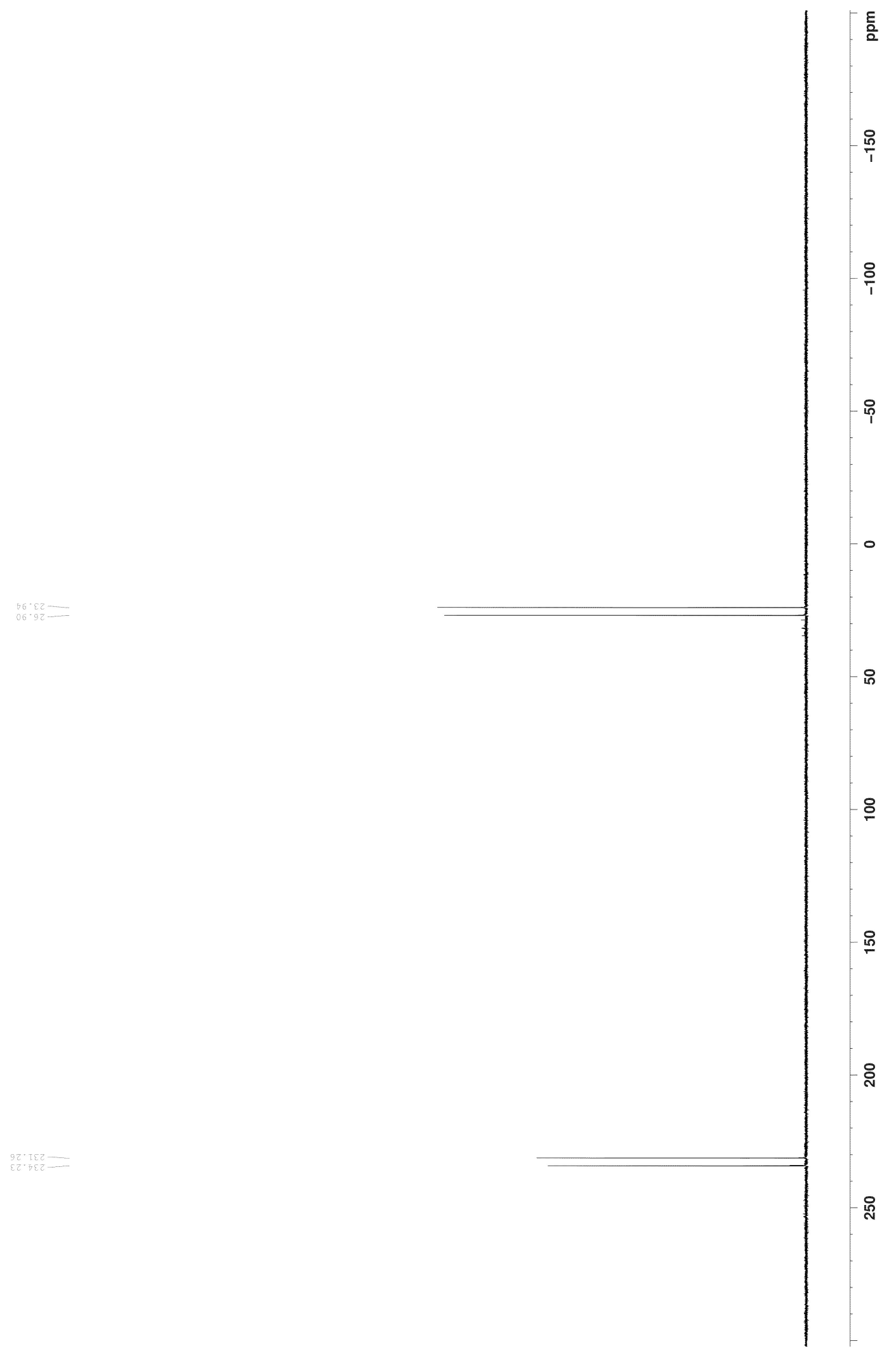

Figure S23. ${ }^{31} \mathrm{P}\left\{{ }^{1} \mathrm{H}\right\}$ NMR spectrum of $\left[\operatorname{IrCl}(\right.$ Eind-PPEP) $](11)\left(\mathrm{THF}-d_{8}, 25{ }^{\circ} \mathrm{C}, 162 \mathrm{MHz}\right)$. 


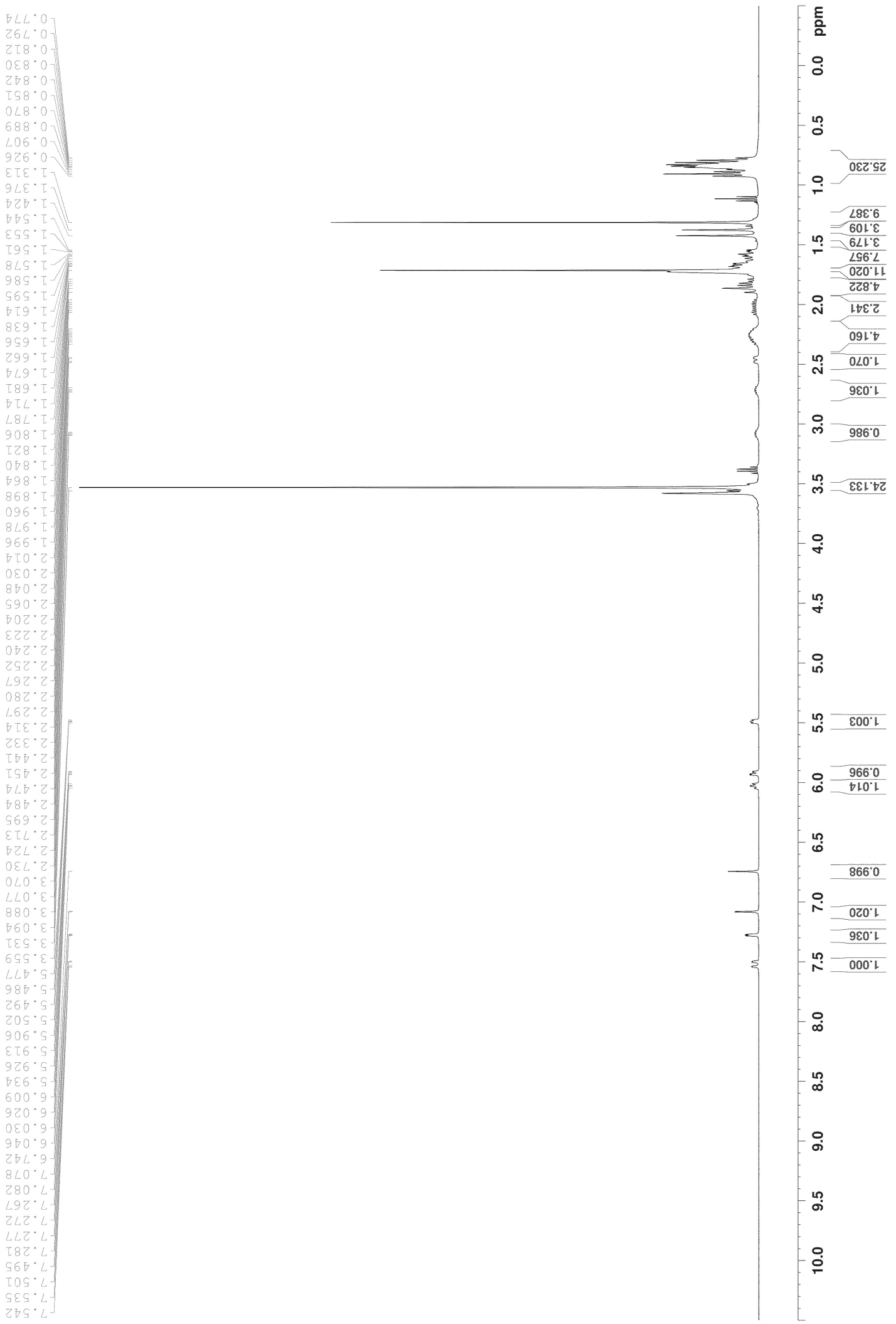

Figure S24. ${ }^{1} \mathrm{H}$ NMR spectrum of $[\mathrm{K}(18 \mathrm{C} 6)][\mathrm{RhCl}($ Eind-PPEP*) $)([\mathrm{K}(18 \mathrm{C} 6)][12])$ (THF- $\left.d_{8}, 25^{\circ} \mathrm{C}, 400 \mathrm{MHz}\right)$. 


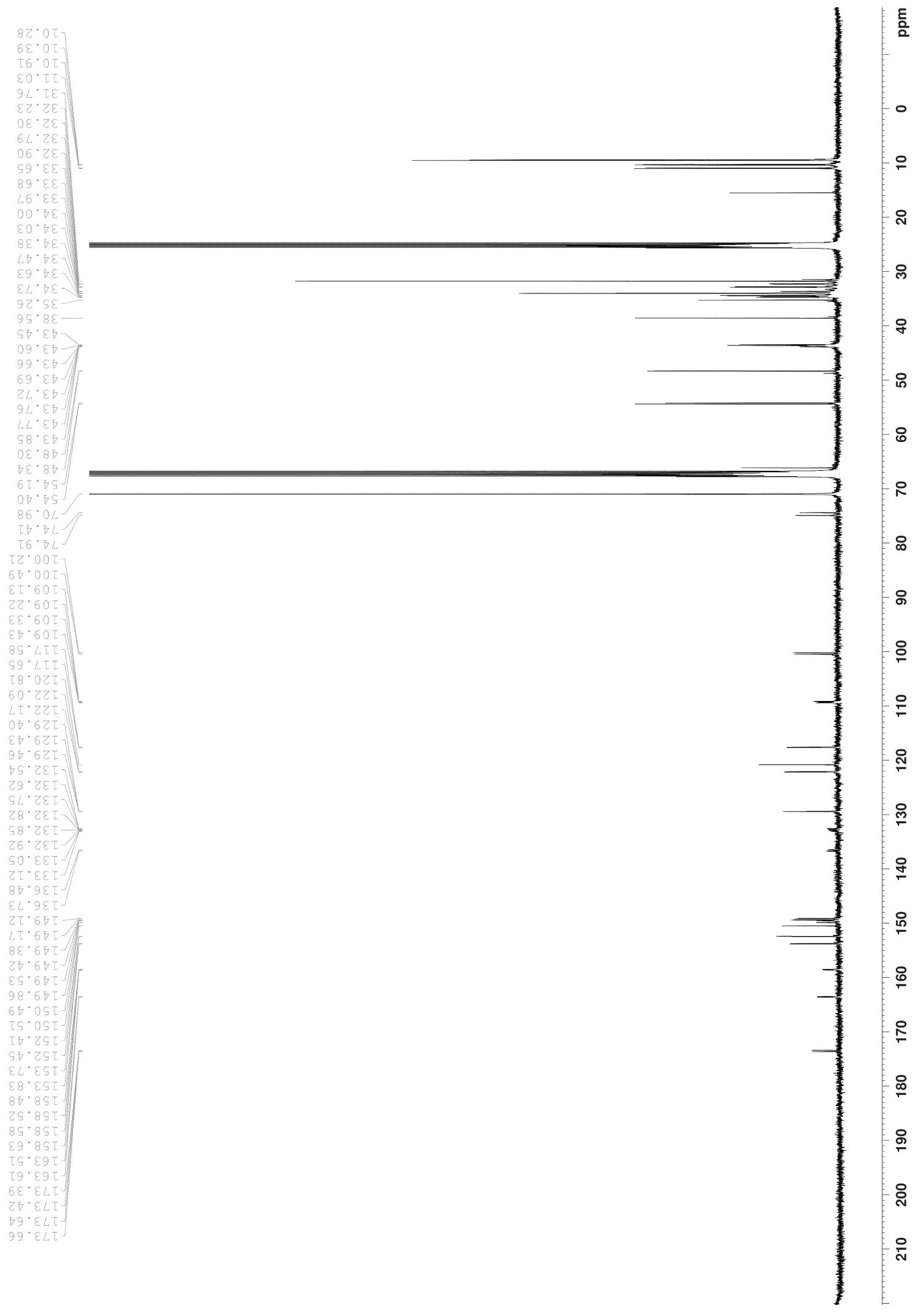

Figure S25. ${ }^{13} \mathrm{C}\left\{{ }^{1} \mathrm{H}\right\}$ NMR spectrum of $[\mathrm{K}(18 \mathrm{C} 6)][\mathrm{RhCl}($ Eind-PPEP* $)]([\mathrm{K}(18 \mathrm{C} 6)][\mathbf{1 2}])$ (THF- $d_{8}, 25^{\circ} \mathrm{C}, 101 \mathrm{MHz}$ ). 


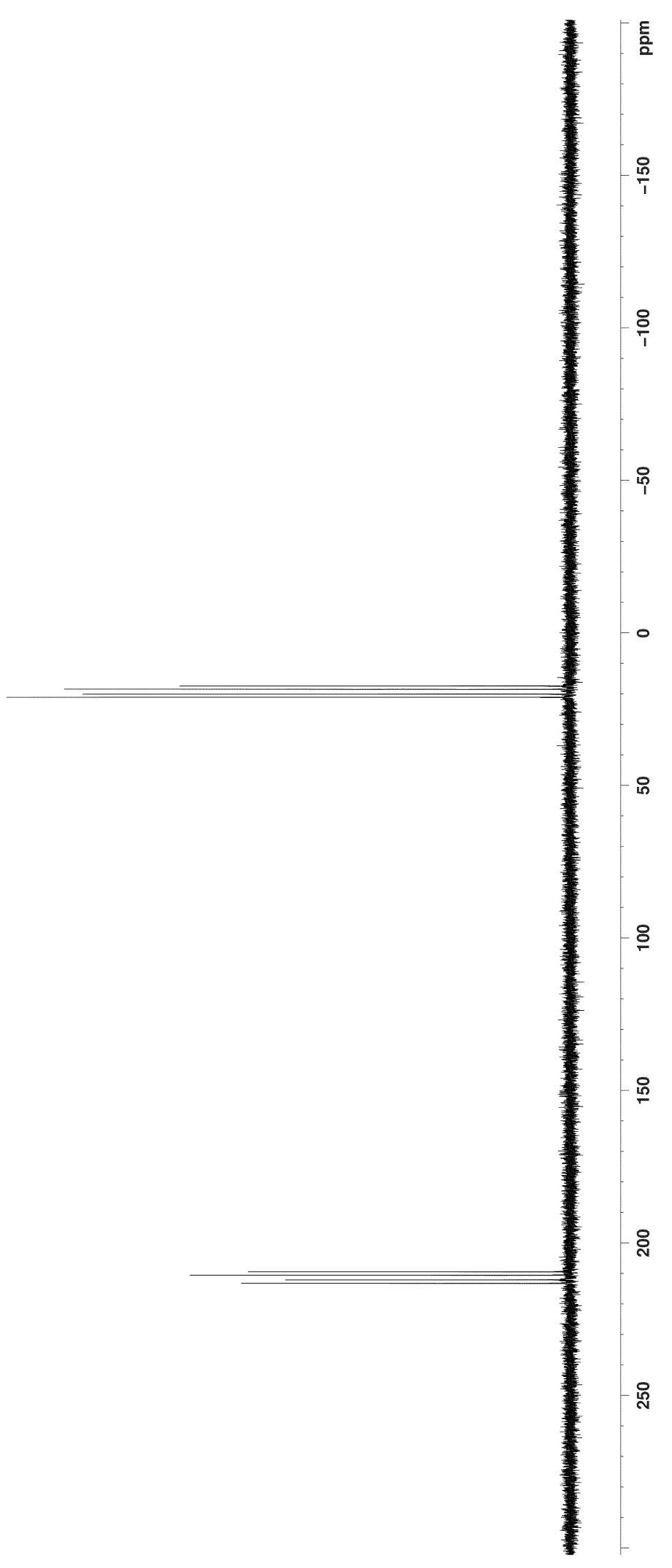

Figure S26. ${ }^{31} \mathrm{P}\left\{{ }^{1} \mathrm{H}\right\}$ NMR spectrum of $[\mathrm{K}(18 \mathrm{C} 6)][\mathrm{RhCl}($ Eind-PPEP*)$)]([\mathrm{K}(18 \mathrm{C} 6)][12])$ (THF- $d_{8}, 25^{\circ} \mathrm{C}, 162 \mathrm{MHz}$ ). 


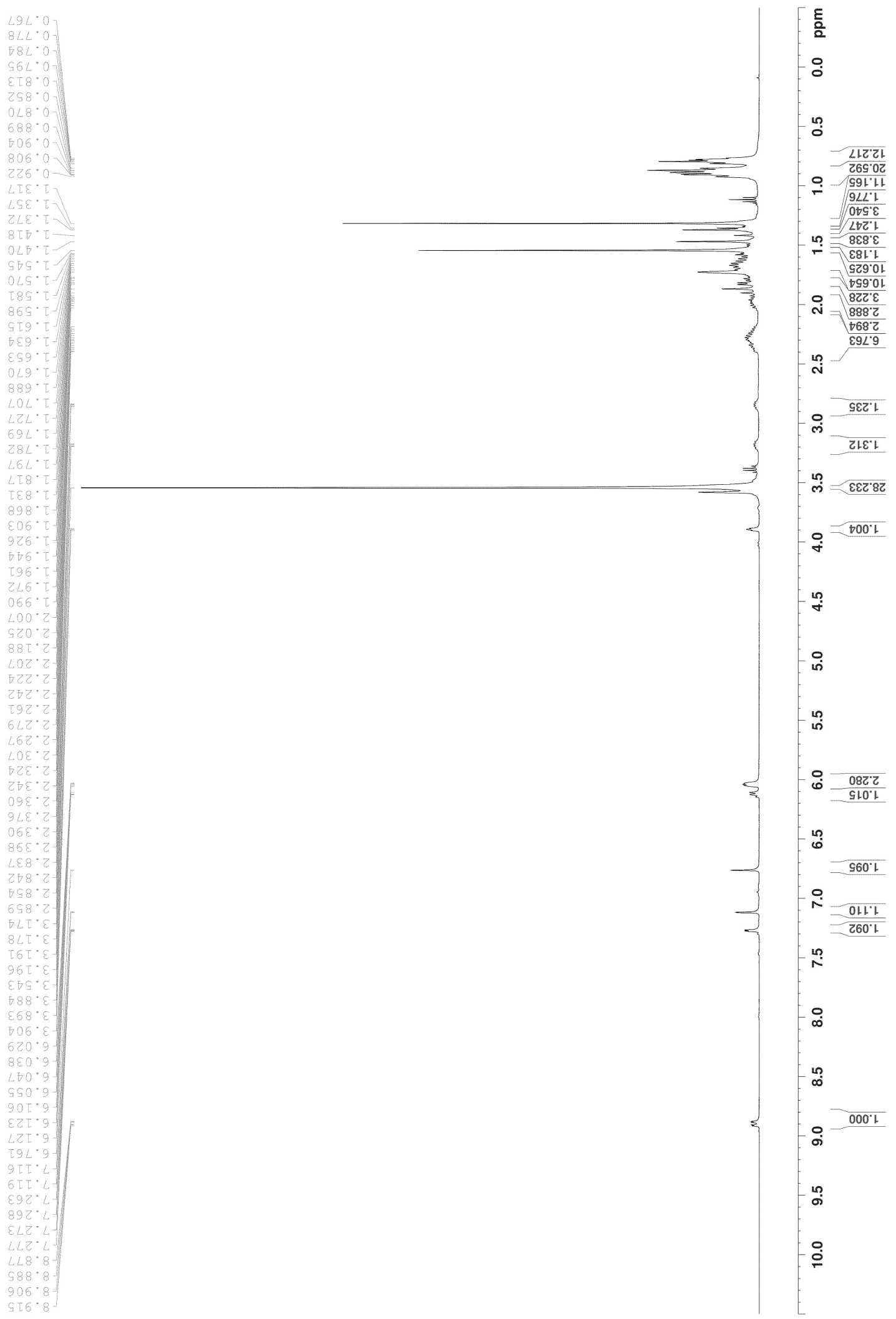

Figure S27. ${ }^{1} \mathrm{H}$ NMR spectrum of $[\mathrm{K}(18 \mathrm{C} 6)][\mathrm{IrCl}($ Eind-PPEP* $)]([\mathrm{K}(18 \mathrm{C} 6)][13])$ (THF- $d_{8}, 25^{\circ} \mathrm{C}, 400 \mathrm{MHz}$ ). 


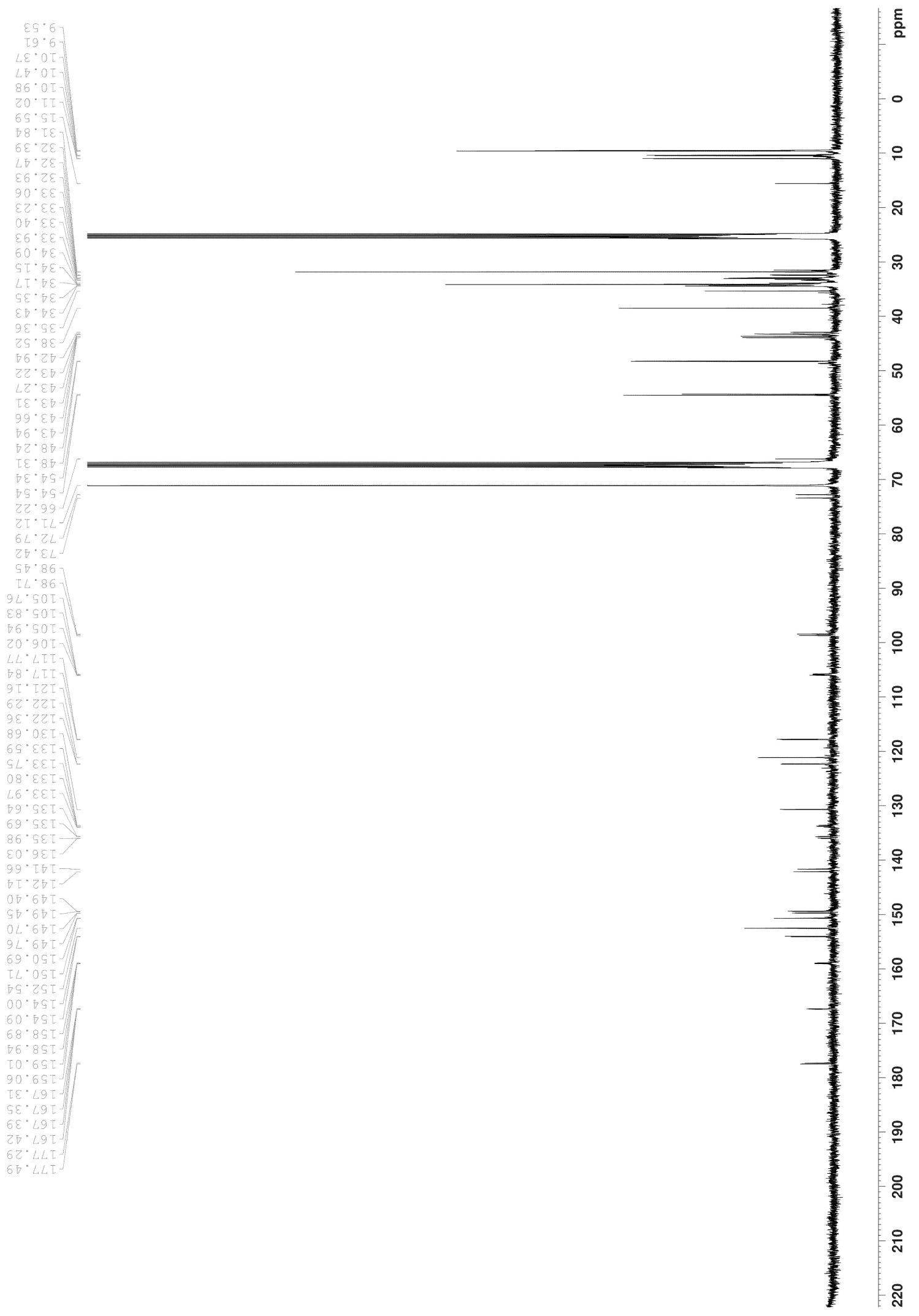

Figure S28. ${ }^{13} \mathrm{C}\left\{{ }^{1} \mathrm{H}\right\}$ NMR spectrum of $[\mathrm{K}(18 \mathrm{C} 6)][\operatorname{IrCl}($ Eind-PPEP* $)]([\mathrm{K}(18 \mathrm{C} 6)][13])$ (THF- $d_{8}, 25^{\circ} \mathrm{C}, 101 \mathrm{MHz}$ ). 


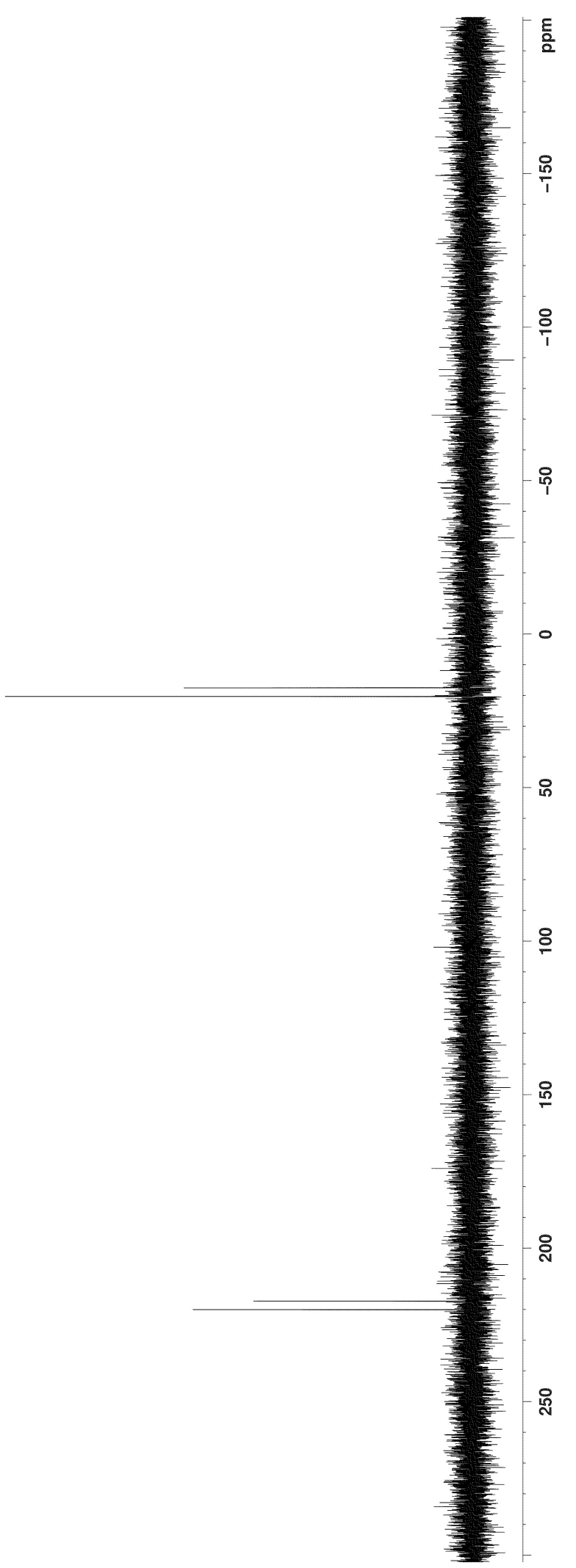

Figure S29. ${ }^{31} \mathrm{P}\left\{{ }^{1} \mathrm{H}\right\}$ NMR spectrum of $[\mathrm{K}(18 \mathrm{C} 6)][\operatorname{IrCl}($ Eind-PPEP* $)]([\mathrm{K}(18 \mathrm{C} 6)][13])$ (THF- $d_{8}, 25^{\circ} \mathrm{C}, 162 \mathrm{MHz}$ ). 

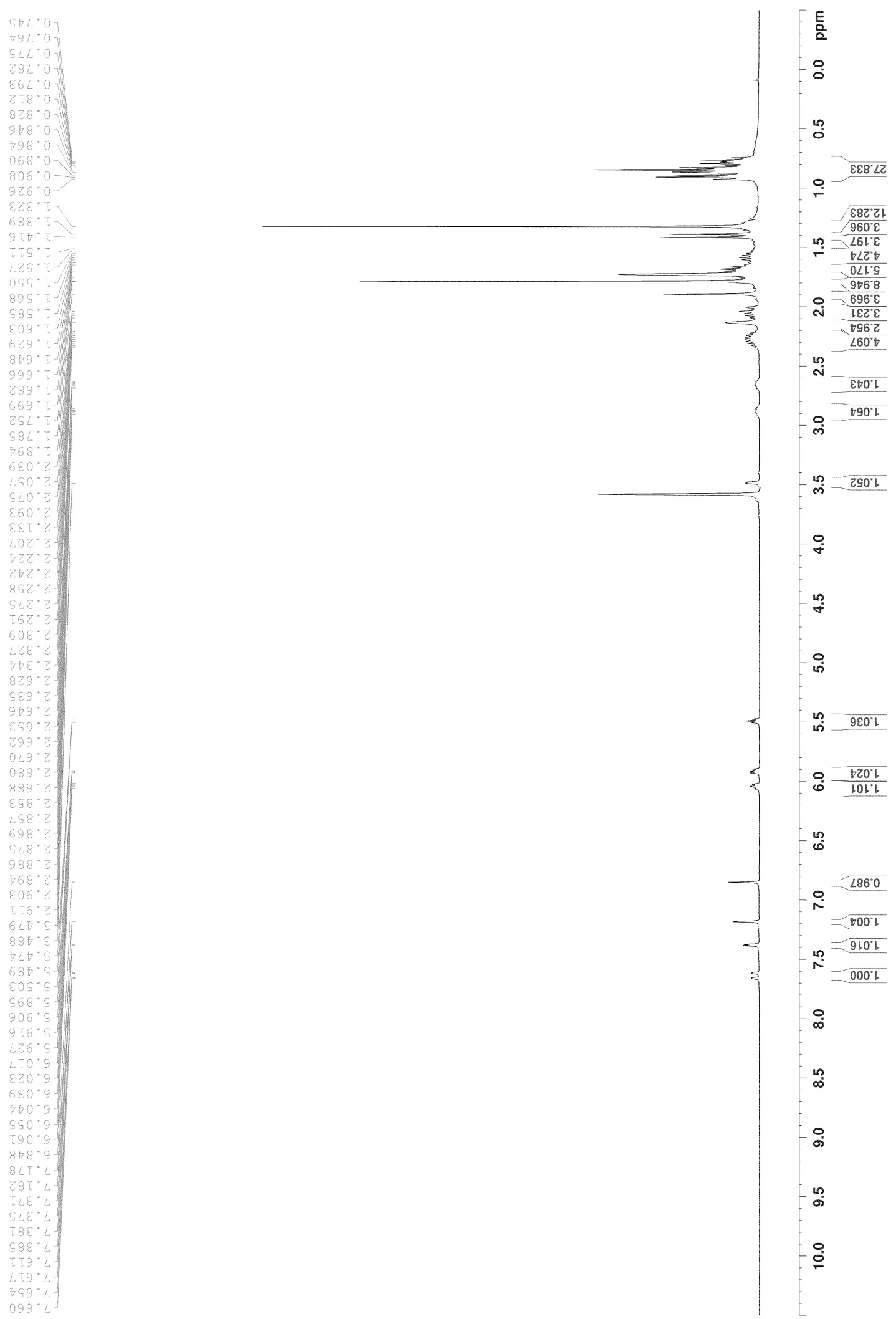

Figure S30. ${ }^{1} \mathrm{H}$ NMR spectrum of $\left[\mathrm{Rh}\left(\mathrm{NH}_{3}\right)(\right.$ Eind-PPEP*) $)$ (14) $\left(\mathrm{THF}-d_{8}, 25{ }^{\circ} \mathrm{C}, 400 \mathrm{MHz}\right.$ ). 


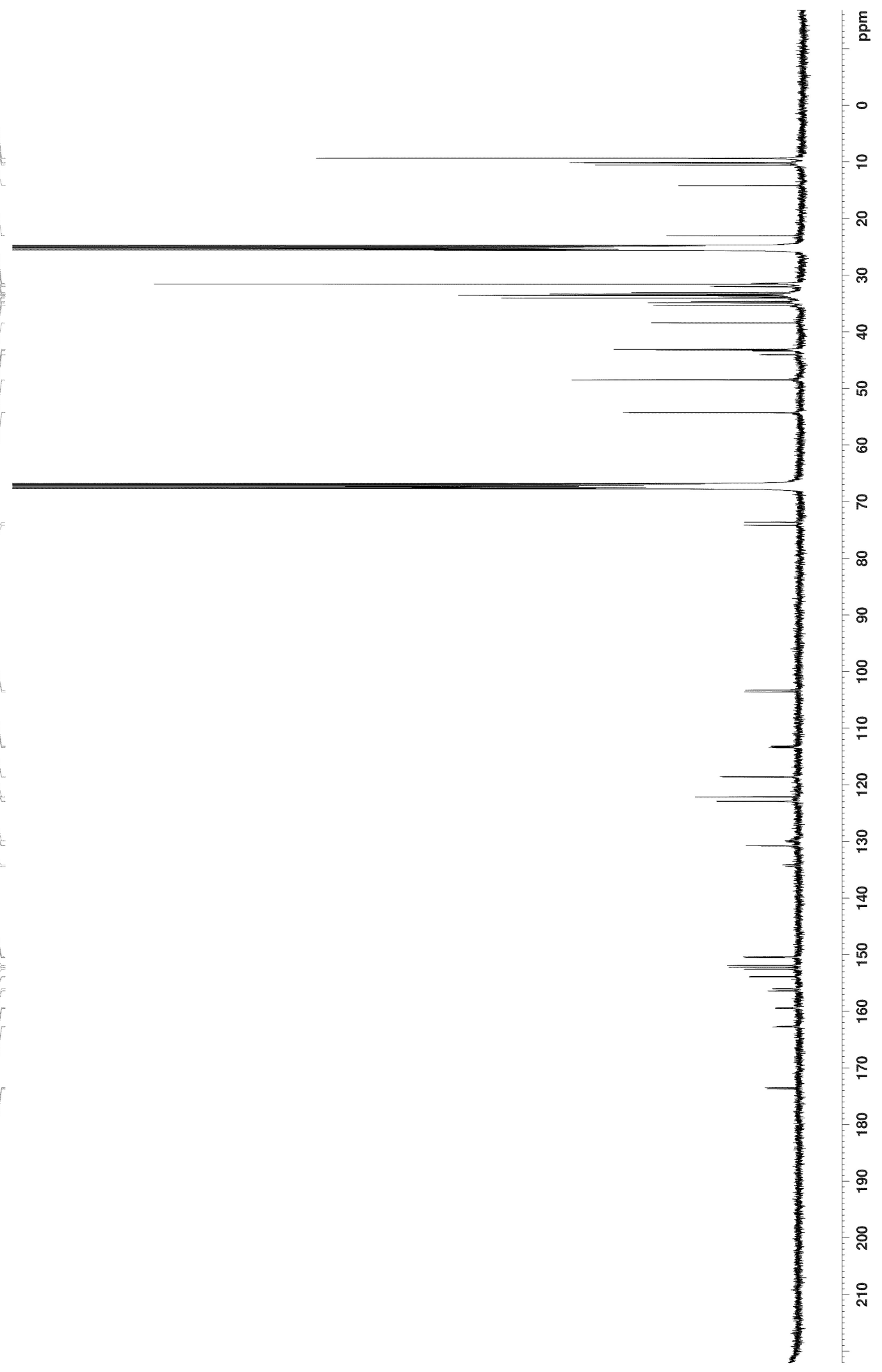

Figure S31. ${ }^{13} \mathrm{C}\left\{{ }^{1} \mathrm{H}\right\} \mathrm{NMR}$ spectrum of $\left[\mathrm{Rh}\left(\mathrm{NH}_{3}\right)(\right.$ Eind-PPEP*)] (14) (THF- $d_{8}, 25^{\circ} \mathrm{C}, 101 \mathrm{MHz}$ ). 




Figure S32. ${ }^{31} \mathrm{P}\left\{{ }^{1} \mathrm{H}\right\}$ NMR spectrum of $\left[\mathrm{Rh}\left(\mathrm{NH}_{3}\right)(\right.$ Eind-PPEP*)] (14) (THF- $d_{8}, 25{ }^{\circ} \mathrm{C}, 162 \mathrm{MHz}$ ). 

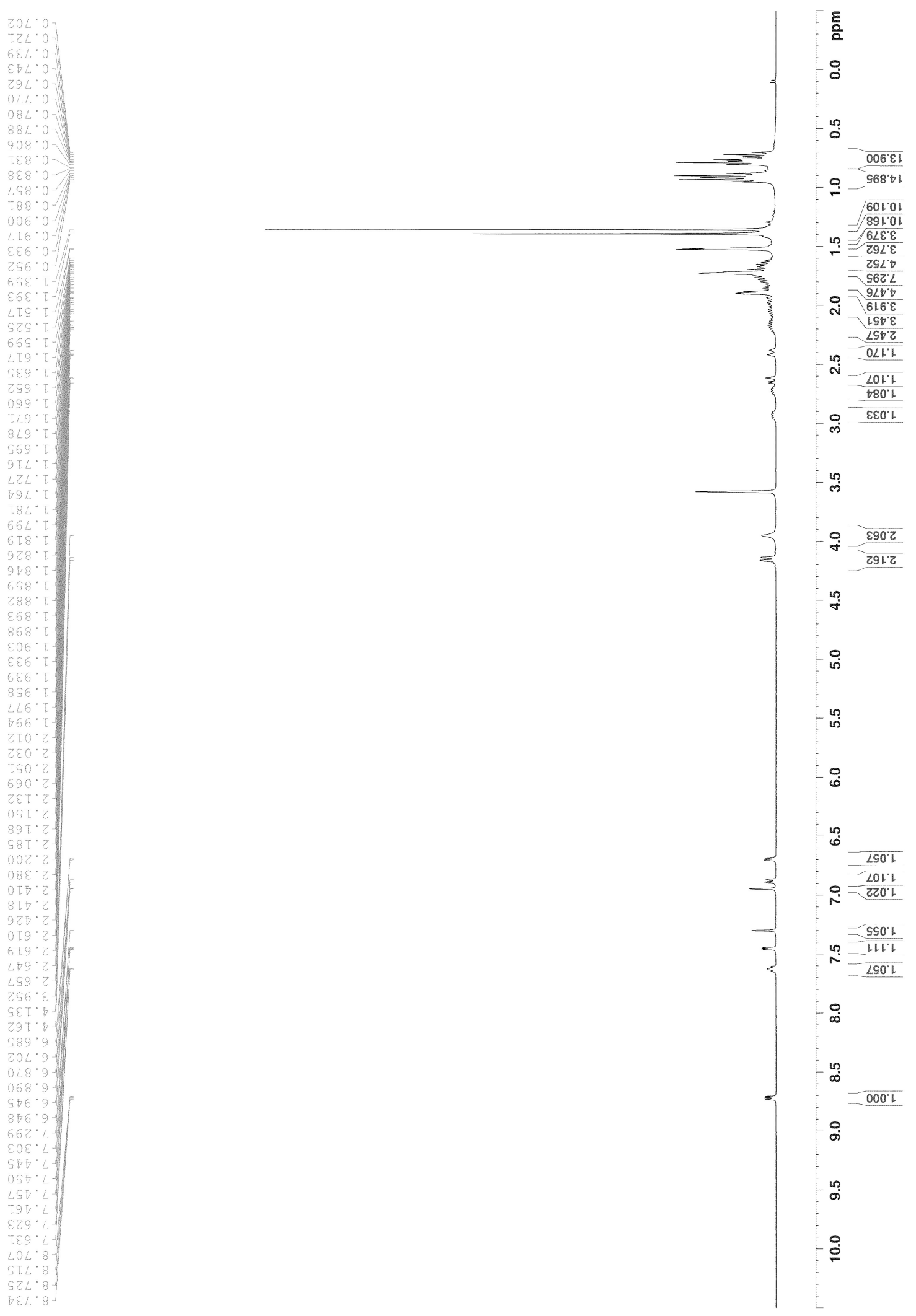

Figure S33. ${ }^{1} \mathrm{H}$ NMR spectrum of $\left[\operatorname{Ir}\left(\mathrm{NH}_{2}\right)(\right.$ Eind-PPEP) $](15)\left(\mathrm{THF}-d_{8}, 25{ }^{\circ} \mathrm{C}, 400 \mathrm{MHz}\right)$. 


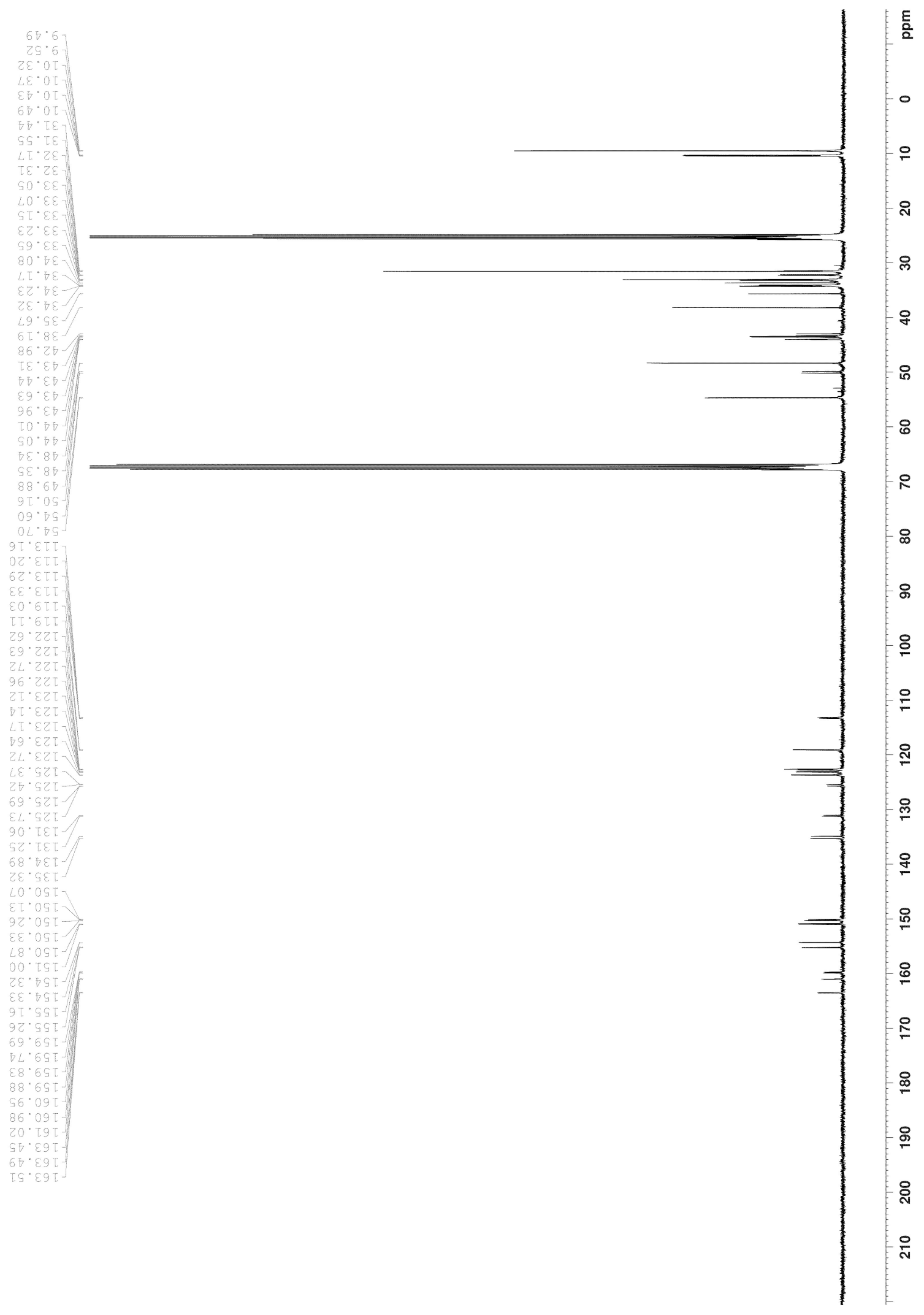

Figure S34. ${ }^{13} \mathrm{C}\left\{{ }^{1} \mathrm{H}\right\}$ NMR spectrum of $\left[\operatorname{Ir}\left(\mathrm{NH}_{2}\right)(\right.$ Eind-PPEP) $](15)\left(\mathrm{THF}-d_{8}, 25{ }^{\circ} \mathrm{C}, 101 \mathrm{MHz}\right)$. 


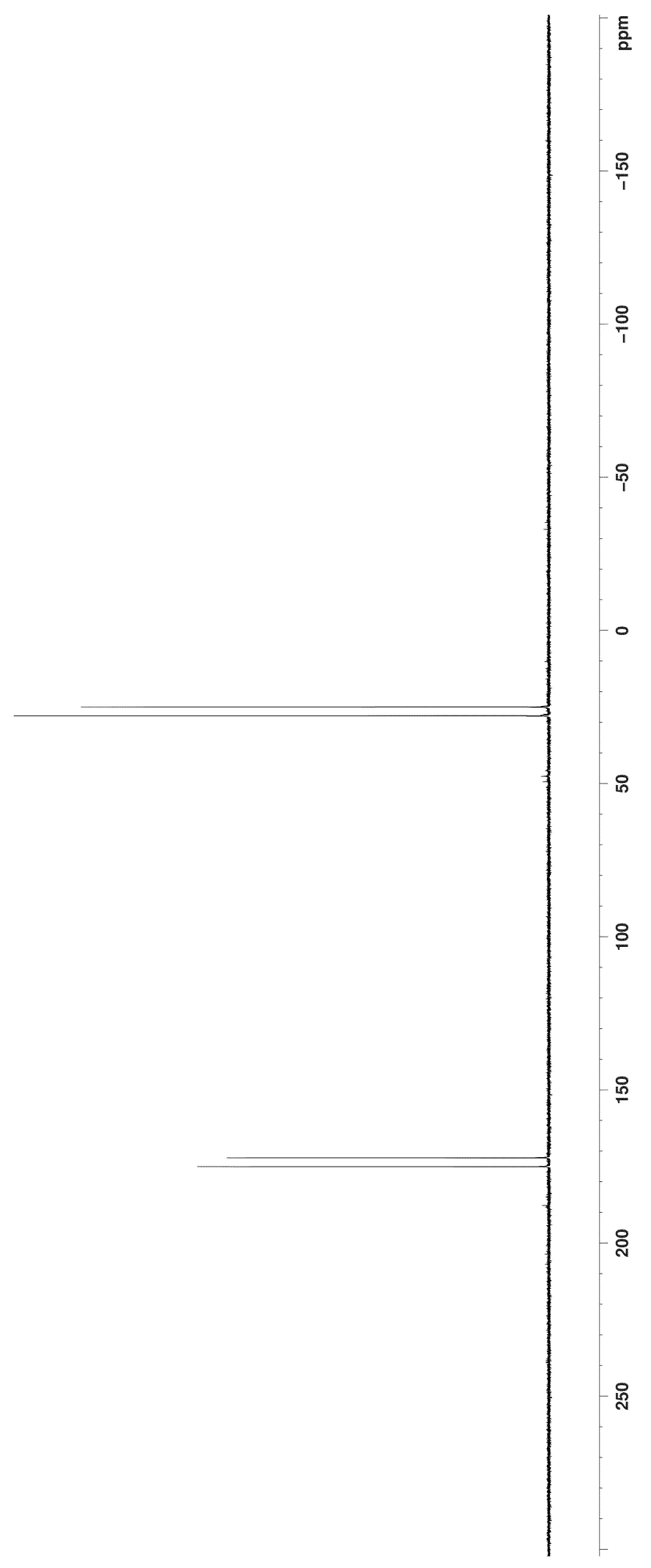

Figure S35. ${ }^{31} \mathrm{P}\left\{{ }^{1} \mathrm{H}\right\}$ NMR spectrum of $\left[\operatorname{Ir}\left(\mathrm{NH}_{2}\right)(\right.$ Eind-PPEP) $](\mathbf{1 5})\left(\mathrm{THF}-d_{8}, 25{ }^{\circ} \mathrm{C}, 162 \mathrm{MHz}\right)$. 